\title{
IIDWBA Algorithm for Integrated Hybrid PON with Wireless Technologies for Next Generation Broadband Access Networks
}

\author{
N. Moradpoor \\ School of Engineering, Computing and Applied Mathematics \\ University of Abertay Dundee, Dundee, UK \\ e-mail: n.moradpoor@abertay.ac.uk
}

\author{
G. Parr, S. McClean, and B. Scotney \\ School of Computing and Information Engineering \\ University of Ulster, Coleraine, UK \\ e-mails: \{gp.parr, si.mcclean, bw.scotney\}@ulster.ac.uk
}

\begin{abstract}
Optical and wireless technology integration has been proposed as one of the most promising nominees for the nextgeneration broadband access networks for quite some time. Integration scheme merges the high-speed and high-capacity of the optical networks with the low-cost, wide-coverage and mobility features of the wireless counterparts for the Subscriber Stations (SSs). It is also financially viable for the telecommunication service providers, particularly in the rural area where the development of optical infrastructure or expansion of the existing telecommunication solutions such as Digital Subscriber Line (DSL), Cable Modem (CM) or T-1/E-1 networks are either too costly or unreachable. In order to successfully integrate the two technologies, there are some technical concerns in terms of Architectural aspects, Physical Layer features and Media Access Control (MAC) related issues, which need to be addressed efficiency and efficiently in order to provide the smooth end-to-end (e2e) integration. This paper is mainly focused upon the analysis of the key topics in MACrelated issues such as $\mathrm{E} 2 \mathrm{E}$ wavelength/bandwidth requests and allocations over the converged scenario. It proposes an Interchannel and Intra-channel Dynamic Wavelength/Bandwidth Allocation (IIDWBA) algorithm where the hybrid Passive Optical Network (PON) acts as a back haul technology for the wireless counterpart. The proposed algorithm works in three phases, namely, Initialisation, Intra-channel bandwidth allocations and Inter-channel bandwidth allocations and is capable of allocating wavelength/bandwidth efficiently and effectively over the converged scenario. Performance of the proposed algorithm is evaluated through conducted simulation scenarios under different simulation parameters, traffic patterns and load values. According to the captured results, IIDWBA algorithm shows a better performance when it is compared to the scenario in which it has not been employed.
\end{abstract}

Keywords: optical and wireless technology integration, Media Access Control (MAC), bandwidth requests and allocations, wavelength allocations

\section{INTRODUCTION}

As current and future applications and services require bandwidth far ahead of the capacity provided by copper-based access technologies such as DSL, CM and T1/E1 networks, subscriber networks based on optical technology have been expanded.

PON, in particular, is the most promising candidate among optical access solutions in terms of maintainability and robustness. PON is a point-to-multi-point (P2MP) fibre to the network premises which can be in the format of the fibre-tothe-home (FTTH), fibre-to-the-PC (FTTPC), fibre-to-the building (FTTB) and fibre-to-the-curb (FTTC). However, FTTC has been considered as the most economical deployment among other FTTX solutions [23]. PON eliminates active components such as multiplexor and demultiplexor from the splitting point and reduces e2e fibre developments.

There have been various efforts on PON multiplexing techniques such as Time Division Multiplexing (TDM), Wavelength Division Multiplexing (WDM) and Code Division Multiplexing (CDM) as well as PON protocols such as Ethernet-PON (EPON) [10], Gigabit-PON (GPON) [11] and Broadband-PON (BPON) [12].

TDM-PON reduces the cost per subscriber by sharing a single wavelength channel with multiple end users. It has inexpensive network components as it requires only one transmitter in the Optical Line Terminal (OLT), no matter how many Optical Network Units (ONUs) are connected, as well as only one type of transmitter in ONUs [23]. However, TDM-PON sacrifices the maximum available bandwidth per subscriber and limits the number of supported subscribers up to 32 [21]. It is also a complicated technique as ONUs are required to be synchronized.

On the other hand, WDM-PON provides multiple wavelength channels with a good security and protocol transparency which offers higher bandwidth and supports more subscribers. It is also a simple technique to implement for the access networks. In WDM-PON, a WDM transmitter, especially in a subscriber side is the most critical component where the associated transmitter should be precisely aligned with the allocated channel [21]. Unlike TDM-PON, in WDMPON, the OLT needs to have an array of transmitters with one transmitter for each ONU. Each ONU also needs to have a wavelength-specific laser. Thus, adding a new ONU can be a problem. Therefore, WDM-PON is less scalable and its components are relatively more expensive than TDM-PON components. The inclusive review of WDM-based PON can be found in [6].

Ethernet-based PON (EPON) appears to be the preferred 
choice among PON standards as Ethernet is a ubiquitous, inexpensive, scalable and off-the-shelf technology. IEEE 802. 3ah [10] specifies the Medium Access Control (MAC) layer characteristics of EPON, which carries the Ethernet frames with $1 \mathrm{~Gb} / \mathrm{s}$ symmetric upstream and downstream transmission speed. IEEE 802. 3av Task Force [26] works on an enhanced version of the IEEE 802. 3h termed $10 \mathrm{~Gb} / \mathrm{s}$ EPON (10 GEPON) in which two solutions have been proposed. A symmetric transmission speed of $10 \mathrm{~Gb} / \mathrm{s}$ upstream and downstream and asymmetric transmission speed of $10 \mathrm{~Gb} / \mathrm{s}$ downstream and $1 \mathrm{~Gb} / \mathrm{s}$ upstream.

PON has been viewed as an attractive solution for the "first/last mile" bandwidth bottleneck problem by providing higher bandwidth with lower costs and supporting subscribers located beyond $20 \mathrm{~km}$ from $\mathrm{CO}$ when it is compared to other copper-based access technologies such as DSL, CM and T1/E1 access networks. However, extending fibre-based infrastructures of PON to the rural area is either too costly or inaccessible. Moreover, PON is unable to provide wireless access services or mobility features for SSs.

World Wide Interoperability for Microwave Access (WiMAX, IEEE 802.16 [27]) standard comes into play as a wireless matching part for PON technology. WiMAX aims to reduce the equipment, operation and maintenance costs and capable of providing the low-cost, wide coverage, fixed and mobile broadband access connections with Quality of Service (QoS) provisioning scheme [28] and [29]. It uses both unlicensed, Industrial and Scientific Medical (ISM), and licensed frequency bands and supports up to $75 \mathrm{Mb} / \mathrm{s}$ data rates with a $20 \mathrm{MHz}$ channel over $3-5 \mathrm{~km}$ distance (under optimal condition). Thus, WiMAX is also suitable for wireless metropolitan area due to providing high bandwidth and supporting large distance. It can work in two modes: point-to-multi-point mode (PMP) and Mesh mode. In PMP mode, there are only communications between Base Stations (BSs) and Subscriber Stations (SSs) while in Mesh mode, the SSs can also communicate with one another. WiMAX also provides wireless access services for rural areas where the development of copper-base technologies or fibre-based broadband are too expensive or inaccessible. However, WiMAX copper-based back haul technologies (DSL and T1/E-1) are the controversial and challenging issues. For instance, DSL performance depends on distance from the Central Office (CO) as well as time of access and T-1/E-1 technology is not a cost-aware and scalable solution.

This is the point where optical and wireless technology integrations come into play where PON can be used as a scalable, cost-aware and potential solution for WiMAX back haul problem whereas WiMAX can extend PON infrastructure to the rural area with relatively lower cost in order to provide wireless broadband services with mobility features for the subscriber stations located in wider distances from $\mathrm{CO}(>>20 \mathrm{~km})$.

In order to successfully integrate the optical and wireless technologies, there are some challenging issues, which need to be addressed efficiently and effectively in order to provide the smooth e2e technology integrations from a given $\mathrm{SS}$ in front-end customer premises all the way up to the OLT in the far-end central office.

For instance, e2e MAC related issues such as e2e QoS provisioning schemes, upstream scheduling or e2e wavelength/bandwidth allocations are the critical issues. For example, e2e wavelength/bandwidth allocations from the OLT in far-end $\mathrm{CO}$ to a given ONU and from a given ONU to the associated BSs and then finally down to the related SSs at the front-end customer premises can be in the following four structures: e2e static wavelength and static bandwidth allocations, e2e static wavelength and dynamic bandwidth allocations, e2e dynamic wavelength and static bandwidth allocations and e2e dynamic wavelength and dynamic bandwidth allocations.

To the best of our knowledge, the traditional single channel TDM-PON has been addressed in most of the existing work related to the optical and wireless integration scenarios.

Using the traditional TDM-PON, where a group of ONUs (typically 16) sharing a single channel, does not seem to be enough when a given ONU is employed as a backhaul for more than a single BS. This is the point where WDM-PON comes into play where multiple wavelengths will be available over a same fibre channel, thus higher bandwidth can be provided by the OLT for a given ONU and more BSs and finally, more number of SSs can be supported.

Providing multiple wavelengths over a single channel in the OLT or in a given ONU carries many challenging issues. For instance, wavelength managements on OLT and ONU, bandwidth managements on OLT and ONU per channel per service cycle, number of ONUs sharing a single channel (static/dynamic), number of BSs sharing a single channel (static/dynamic), channel allocation de-allocation and reallocations are some of the challenging issues.

From the above observations and discussions, we are motivated to combine the wavelength routing and highcapacity of WDM-PON, power-splitting and lower-cost of TDM-PON with high coverage and mobility features of the wireless counterpart for the optical and wireless integration scenario.

In order to provide the full dynamic wavelength/bandwidth allocations across hybrid PON integration with wireless technology, an Inter-channel and Intra-channel Dynamic Wavelength/Bandwidth Allocation (IIDWBA) algorithm is proposed. In IIDWBA, excess bandwidth from all the available channels can be collected per service cycle and passed to a central scheduler termed global scheduler in order to schedule, allocate and then distribute among the channels which are not satisfied by the minimum guaranteed bandwidth.

The remainder of this paper is prepared as follows. In Section II, the existing work related to the optical and wireless technology integrations is briefly discussed, which is mainly focused on the MAC-related issues of the converged scenarios. The e2e wavelength/bandwidth allocations over the converged scenario are detailed in Section III. The proposed 
Inter-channel and Intra-channel Dynamic Wavelength/Bandwidth Allocation (IIDWBA) algorithm is fully discussed in Section IV. Sections V and VI include the implementations and the captured results, respectively followed by the conclusions and references in Section VII and VIII, both correspondingly.

\section{RELATED WORK}

To date, a wide range of research has been done for the successful integration of the optical and wireless technologies. The converged network saves the Capital Expenditures (CapEx) and Operating Expenses (OpEx) costs and provides reliable and robust connections for the next-generation broadband access networks.

The integrated scenario has been considered under three categories of: Architectural, Physical layer and MAC layer issues. The Architectural aspects [3], [7], [16], [17], [31], [32], [34] and [37] include the way two technologies connect to each other along with the e2e functional modules, physical structure, component specifications as well as the distance arrangements. In Physical layer issues, most of the works were focused on providing the cost-effective and reliable Radio over Fibre (RoF) systems, for instance, providing RoF systems in order to use a single modulator for both wired and wireless services simultaneously [19]. The MAC layer problems include the $\mathrm{e} 2 \mathrm{e}$ resource negotiations, resource allocations and resource scheduling such as wavelength/bandwidth requests and allocations, upstream scheduling techniques as well as the e2e QoS supports.

As the research in this paper is related to the MAC-related issues of the converged scenario, the previous works related to this aspect is selected and discussed as follows.

The MAC-related issues for the integrated scenario were discussed for the first time in [7]. The authors raised several issues for the bandwidth allocations, packet scheduling, QoS support and user mobility issues, e.g. handover operations, for the converged scenario. In [30], authors investigated the scheduling techniques in hybrid optical and wireless access networks aimed to improve the performance and guarantee the QoS for different class of services. The proposed centralized scheduling provided the better performance when it was compared to the distributed scheduling (multi-hob scheduling) in terms of delay for all service classes as well as the throughput. However, no QoS mapping mechanism or bandwidth scheduling was discussed. In [3], authors proposed a slotted-DBA (S-DBA) algorithm for the integrated passive optical and IEEE 802.16 networks, which aimed to increase the bandwidth utilizations by reducing the signaling overhead caused by the cascading bandwidth requests and grants in both optical and wireless parts of the converged scenario. In [33], authors proposed an intra ONU-BS scheduling algorithm termed Hybrid Priority Weighted Fair Scheduling (HPWFS) in order to progress the QoS performance of the EPON and WiMAX integrations without bandwidth starvations for the lower-priority class of services. HPWFS worked along with the Prediction based executive DBA (PE-DBA) algorithm and QoS mapping mechanism in order to improve the QoS performance of all traffic classes in EPON and WiMAX networks. In [34], authors proposed a DBA algorithm for the suggested Optical-Optical-Wireless (OOW) architecture. The proposed DBA algorithm was executed in three levels of WiMAX BS, integrated ONU and sub-OLTs. The sub-OLTs connected to OLT in a point-to-multipoint manner, thus, OLT did not need to run a bandwidth allocation algorithm. The simulation experiments showed the improvements in average throughput and average and maximum delay for different classes of services. In [20], the authors of this paper investigated the possible challenging issues for the integrated structures of the TDM-PON and WDM-PON with WiMAX and Wireless Fidelity (Wi-Fi) networks. To reduce the e2e delay and provide the QoS for diverse service classes, the six existing upstream scheduling mechanisms were compared in two levels, which were distributed on Access Points (APs) from Wi-Fi domain and BSs from WiMAX domain. Performance evaluations of the existing scheduling techniques for three popular service classes (Quad-play) were studied, which showed the strong impact of using an efficient up-link scheduler in converged scenario.

For more work related to the MAC-Layer aspects of the optical and wireless converged scenarios we refer the readers to [15], [18] [31], [35] and [36]. As the work in this paper is also related to the bandwidth allocation techniques in PONs, which is employed in order to backhaul wireless technologies, we would like to refer the readers to [1], [4], [8], [13], [22], [24] and [25] for bandwidth allocation issues over PONs.

To the best of our knowledge, TDM-PON has been addressed in most of the existing work related to the optical and wireless converged scenarios.

In conventional TDM-PON, the $1 \mathrm{~Gb} / \mathrm{s}$ symmetric channel capacity is shared by group of ONUs (typically 16) each supplied with $\sim 65 \mathrm{Mb} / \mathrm{s}$ of channel capacity $(1 \mathrm{~Gb} / \mathrm{s}$ divided by16) which almost matches the total capacity offered by an IEEE 802.16 BS which is about 70 Mbps over a $20 \mathrm{MHz}$ channel [7]. Therefore, providing services to a single BS by a single ONU from a conventional TDM-PON is feasible as the total channel capacity will be almost matched. Thus, there will be no bandwidth bottleneck problem at the converged point (ONU-BS). However, the number of BSs supported by a single ONU is limited to one BS and total number of supported BSs for the whole converged scenario is limited to 16 BSs $(1 \mathrm{BS} \times 16$ ONUs $)$.

A solution for such a problem is to provide the OLT in CO with multiple wavelengths over the same fibre channel which resembles the conventional WDM-PON structure. OLT with multiple channels provides higher bandwidth for each ONU, which results in supporting more BSs and finally, more SSs at the front-end customer premises. For instance, a given OLT, which is associated with 16 ONUs with four supported wavelength channels, can provide bandwidth up to $260 \mathrm{Mb} / \mathrm{s}$ $(65 \mathrm{Mb} / \mathrm{s} \times 4$ channels) per ONU. If $260 \mathrm{Mb} / \mathrm{s}$ bandwidth is available per ONU, each ONU can support up to 4 BSs which increases the total number of supported BSs for the whole converged scenario to 64 (16 ONUs $\times 4$ BSs).

However, providing OLT and ONU with multiple wavelengths brings many challenging issues. For instance, 
OLT-ONU and ONU-BS real-time and dynamic wavelength and bandwidth allocation techniques, light source requirements on OLT, ONUs and BSs, number of ONUs and BSs allocated per channel per service cycle are some of the challenging issues. For example, number of ONUs and BSs which is allocated per channel per service cycle can be fixed in static allocations or flexible in dynamic allocations. In dynamic allocations, it will be changed per service cycle according to the channel load, total network traffic or the QoS requirements per class of services.

The major contributions in this paper are as follows.

- In terms of MAC-related issues, an Inter-channel and Intra-channel Dynamic Wavelength/Bandwidth Allocation (IIDWBA) algorithm is proposed in order to allocate OLT's available wavelengths/bandwidth among ONUs as well as ONU's available wavelengths/bandwidth among the associated BSs in a dynamic manner during each service cycle. The IIDWBA algorithm is implemented in two places: OLT in $\mathrm{CO}$ and ONU near BSs in order to provide the full distributed and dynamic wavelength/bandwidth allocations across OLT - ONUs as well as ONU - BSs. While a given DBA algorithm deals with bandwidth allocations inside a given channel, IIDWBA algorithm works on top of a given DBA algorithm inside the OLT and ONU in association with multiple channels. The proposed algorithm in this paper is evaluated in terms of different QoS metrics, which shows a better performance when it is compared with the existing work.

- The full Multipoint Control Protocol Extension (MPCP Ext.) is also implemented, which works in context of the converged structure and provides e2e communications such as OLT-ONU and ONU-BS auto-discovery, registrations and wavelength/bandwidth allocations.

\section{HIERARCHICAL WAVELENGTHS AND BANDWIDTH ALLOCATIONS IN CONVERGED ARCHITECTURE}

This Section briefly discusses how the wavelength/bandwidth is hierarchically allocated in this paper from the OLT in CO to the ONUs and then from the ONUs down to the associated BSs.

In the converged network model in this paper, the OLTONUs wavelength/bandwidth allocations as well as ONU-BSs wavelength/bandwidth allocations are fully dynamic. Therefore, a Dynamic Wavelength Allocation (DWA) algorithm and a Dynamic Bandwidth Allocation (DBA) algorithm are required inside the OLT and ONUs in order to allocate wavelengths (channels) as well as bandwidth (portion of a given channel), correspondingly from the OLT to ONUs and also from a given ONU to the associated BSs in an efficient and effective way. The DWA and DBA decisions will be made based on the ONU/BS actual need (immediate queue status), OLT/ONU available wavelength/bandwidth; OLT/ONU/BS waveband boundaries as well as the actual traffic load on each channel.

In the converged network model in this paper, MPCP
Extension (MPCP Ext.) [2] is a dominant protocol for the communications over the converged scenario and includes the auto-discovery and registration processes for the ONUs/BSs as well as the wavelength/bandwidth allocation acknowledgments which are the outputs from the DWA and DBA algorithms. MPCP was developed by IEEE 802.3ah [10] in order to settle the real-time bandwidth negotiations in the TDM-based PON. It includes five 64 MAC messages embracing both auto-discovery and bandwidth request/grant messages termed REGISTER_REQUEST, REGISTER, REGISTER_ACKNOWLEDGE, REPORT and GATE messages, Figure 1. While the MPCP provides time-slot assignment features, MPCP Ext. [2] provides the wavelength allocation aspects inside the conventional MPCP. MPCP Ext. enables the OLT to schedule transmission to and reception from a given ONU on any wavelength channel supported by the OLT and ONU [1]. MPCP Ext. carries the decisions of the both DWA and DBA algorithms between the OLT and ONUs as well as the ONU and BSs inside a single IEEE 802.3ah Ext. GATE message. During the auto-discovery and registration processes, which take place over the original wavelength channel of the TDM-based PONs, the newly joined ONUs/BSs indicate information to help OLT/ONU to identify ONUs/BSs WDM architecture, respectively, Table.1. This information will be collected from the REGISTER_REQUEST MPCPDU of the MPCP Ext. message received from a given ONU/BS on the OLT/ONU, both respectively. The OLT/ONU then grants wavelength/bandwidth to a given ONU/BS, respectively, by employing the implemented wavelength/bandwidth allocation algorithms. The allocated channel id along with the allocated bandwidth, transmission start time and transmission durations will be occupied inside the GATE messages which will be then broadcasted to the ONUs/BSs over the converged network.

During a given service cycle, DWA algorithm decides which of the available upstream channels should be allocated while DBA algorithm decides what portion of the allocated channel along with the transmission start time and transmission duration must be assigned to a given ONU/BS. Therefore, when $\mathrm{ONU}_{\mathrm{i}} / \mathrm{BS}_{\mathrm{i}}$ receives $\mathrm{GATE}_{\mathrm{j}}$ message on Cycle $_{\mathrm{n}}$, it starts sending traffic on channel $k$ (the allocated channel), after $\alpha \mathrm{ms}$ (transmission start time) for $\beta \mathrm{ms}$ duration (transmission duration). $k, \alpha$ and $\beta$ are specified by the DWA and DBA algorithms, then filled inside the GATE $_{j}$ message and finally broadcast to all the ONUs/BSs.

In this paper, an Inter-channel and Intra-channel Dynamic Wavelength/Bandwidth Allocation (IIDWBA) algorithm is proposed in order to allocate the available and supported wavelength/bandwidth from the OLT to ONUs and from a given ONU to the associated BSs in an efficient and effective manner. While a given DBA algorithm deals with the dynamic bandwidth allocation techniques inside a single channel, IIDWBA algorithm works on top of a given DBA algorithm and deals with multiple channels. The IIDWBA algorithm has a global and real-time knowledge about the local DBA on each single channel thus it provides a better system performance when it is compared with a DBA over a 
single channel.

The next Section discusses the proposed IIDWBA algorithm and its functionalities as follows.

\section{AN INTER-CHANNEL AND INTRA-CHANNEL DYNAMIC WAVELENGTH/BANDWIDTH ALLOCATION (IIDWBA) ALGORITHM}

The IIDWBA algorithm, which is implemented inside the OLT and ONUs, works in three different phases termed Initialisation, Intra-channel bandwidth allocations and Interchannel bandwidth allocations.

In order to save space and avoid repetitions, a given server station is termed as SST, which can be the OLT or ONU and a given client station is termed as CS, which can be the ONU or BS.

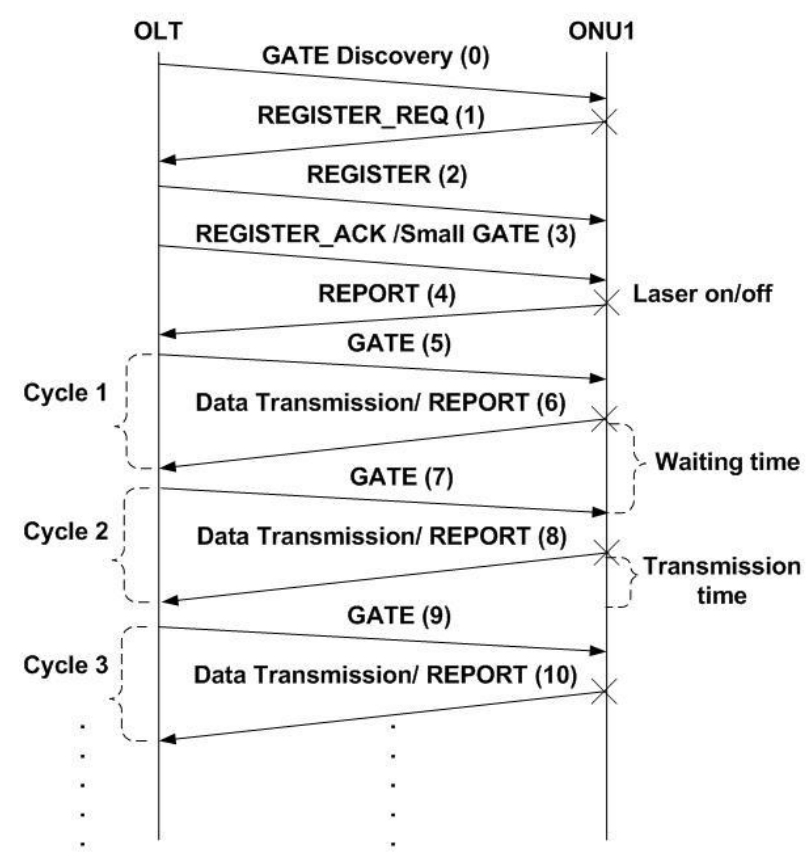

Figure 1 MPCP Resource Negotiations

Table.1 MPCP Ext. [2] in context of the converged scenario

\begin{tabular}{|l|l|l|}
\hline Name & Number of Bits & Values \\
\hline $\begin{array}{l}\text { transmitter and receiver } \\
\text { type }\end{array}$ & 2 bits each & $\begin{array}{l}\text { no WDM (0), fixed- } \\
\text { tuned (1), tunable } \\
(2) \text { and reserved (3) }\end{array}$ \\
\hline $\begin{array}{l}\text { transmitter and receiver } \\
\text { tuning time }\end{array}$ & 16 bits each & $\begin{array}{l}\text { integer multiple of } \\
\text { unit time (e.g. 2 for } \\
2 \text { microsecond) }\end{array}$ \\
\hline wavelength id type & 1 bit & $\begin{array}{l}\text { two-level hieratical } \\
\text { encoding (0) and flat } \\
\text { encoding (1) }\end{array}$ \\
\hline $\begin{array}{l}\text { transmitter and receiver } \\
\text { wavelength band }\end{array}$ & 4 bits each N/A \\
\hline $\begin{array}{l}\text { transmitter and receiver } \\
\text { supported wavelengths }\end{array}$ & $\begin{array}{l}16 \text { bits each If } \\
\text { wavelength_id_type }=0 \\
\text { wavelength_id_type }=1\end{array}$ & N/A if \\
\hline
\end{tabular}

An SST is a network component that provides bandwidth/wavelength or generally services for a given CS periodically, and a given CS is a network component that asks for resources and services from a given SST in a regular manner. At any time, a given SST can be a CS, and a given CS can be an SST. For instance, in the converged scenario in this paper, a given ONU is a CS when it asks for wavelength/bandwidth from the OLT and is a SST when it provides wavelength/bandwidth for a given BS. Moreover, a given BS is a CS when it asks for wavelength/bandwidth from the associated ONU and an SST when it provides services for the associated wireless SSs. However, the OLT at the far-end $\mathrm{CO}$ and the SSs at the front-end customer premises are the ultimate SST and CSs, respectively.

Three phases of the IIDWBA algorithm, which are developed on all the SSTs, particularly the OLT and the ONU, along with the algorithms and flowcharts are as follows.

\section{A. Initialisation phase}

The Initialisation phase, which is the phase one of the proposed IIDWBA algorithm, includes the auto-discovery and registration processes during which the CSs $(\mathrm{ONUs} / \mathrm{BSs})$ join the converged network, and a wavelength will be assigned to each of them.

Based on the number of the CSs in an optical domain of a given SST as well as the supported and available wavelengths on a given SST/Cs, multiple CSs can share a single channel during the phase one. Number of the CSs sharing a single channel can be changed by next phases of the IIDWBA algorithm when the network traffic builds up.

The Initialisation phase is implemented on all the SSTs and runs before a given CS receives permission from the associated SST in order to access to any available channel (bandwidth allocations). During the phase one of the IIDWBA algorithm, a given CS will be allocated with a wavelength which helps it to send the first REPORT message (buffer status) towards the associated SST. All the autodiscovery and registration processes among CSs and a given SST occur over the negotiation channel, $w_{0}$, which is separated from data channels, $w_{i=1, \ldots n}$.

An algorithm and a flowchart, Figure 2, are developed for the Initialisation phase of the IIDWBA algorithm as follows.

\section{Initialisation phase: algorithm and flowchart}

\section{Given:}

1) $\alpha$ :Total number of the supported wavelengths on a given SST.

2) $\beta$ : Total number of the CSs in a given SST's domain.

\section{Define:}

1) $\mu:$ Average number of the CSs per channel for a given SST.

$$
\mu=\beta / \alpha
$$




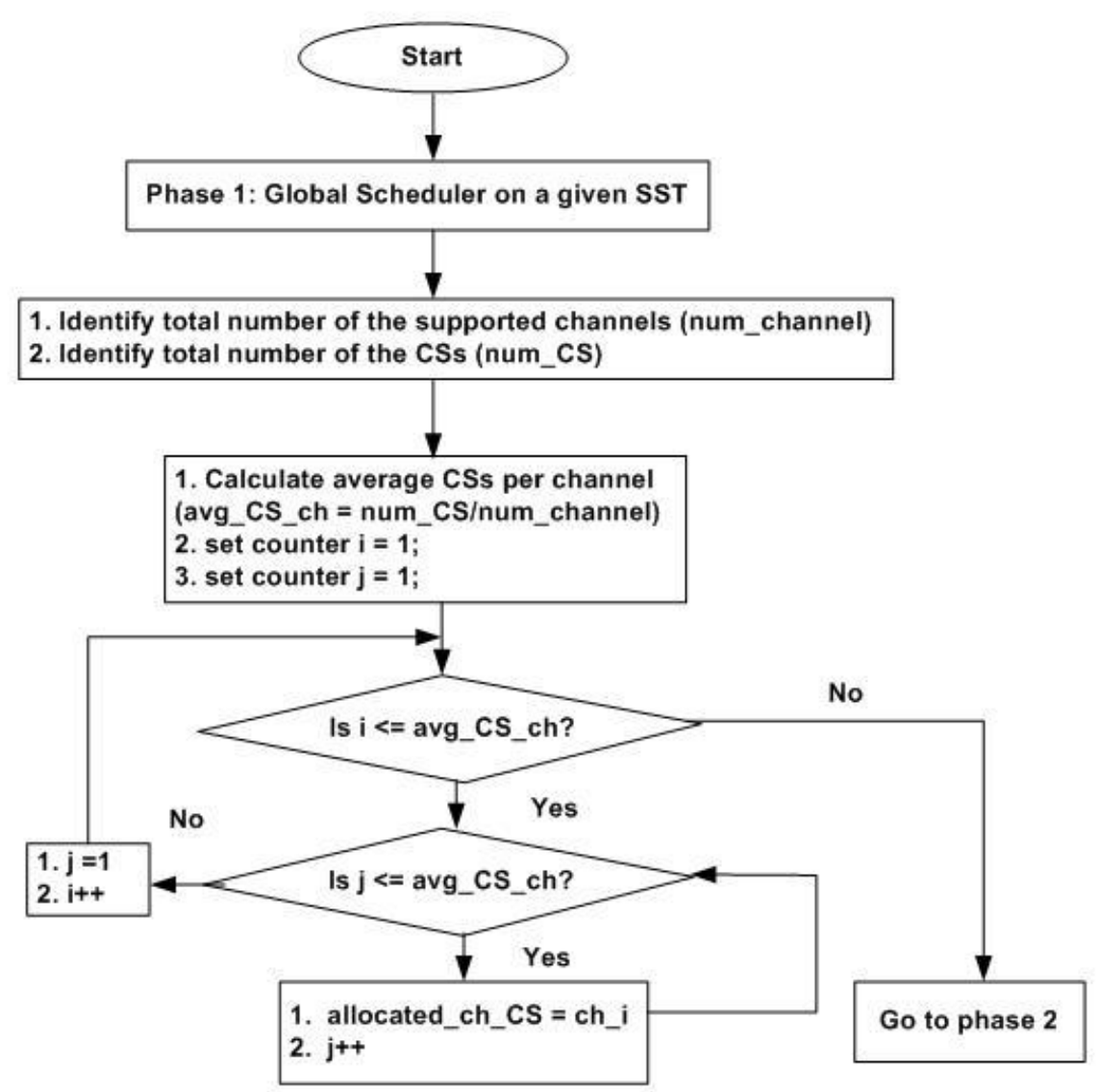

Figure 2 Flowchart for Initialisation phase of the IIDWBA algorithm

This is a default value which is calculated by each SST at the network setup stage. For simplicity, it is assumed that $\alpha$ and $\beta$ both are even numbers.

2) $N_{C S}$ : Number of the CSs per channel on a given SST.

Total number of the CSs per channel on a given SST will be changed during the next two phases of the IIDWBA algorithm. In order to avoid a single heavily loaded CS monopolising the whole channel, $N_{C S}$ is restricted to the average number of the CSs per channel and the total number of the CSs on a given SST's domain therefore:

$$
\mu \leq N_{C S} \leq \beta
$$

The associated flowchart for the Initialisation phase of the IIDWBA algorithm is represented in Figure 2.

As it is shown in Figure 2, the Initialisation phase of the IIDWBA algorithm first identifies the total number of the supported channels as well as the total number of the CSs associated per SST over the converged scenario. The average number of the CSs per channel will be calculated next. Then it starts randomly allocating the channel IDs to all the CSs in such an order that the number of the CSs per channel on a given SST will be the same. Finally, the allocated channel IDs will be acknowledged to all the CSs associated to a given
SST.

Generally speaking, the allocated channel IDs are the default channels which will be granted in order to let a given CS sending the first group of the related data traffic or the first MPCP Ext. REPORT messages to the associated SST. The number of the CSs sharing a single channel, which is the same in this stage, will be changed dynamically during phase three of the IIDWBA algorithm based upon the load on the CSs. This will be discussed later.

\section{Summary of the general function for Initialisation phase (phase one) of the IIDWBA algorithm on a given SST:}

1) Identifying the average number of the CSs per channel on each SST.

2) Assigning a default channel ID to all the CSs associated with a given SST.

3) Finishing the auto-discovery and the registration processes for each CS and receiving the first associated queue status (REPORT message).

The next section provides a detailed discussion for the phase two of the IIDWBA algorithm as follows. 


\section{B. Intra-channel bandwidth allocations phase}

Phase two of the IIDWBA algorithm, which is termed as Intra-channel bandwidth allocations phase, will be executed immediately after the phase one and will be accomplished once per service cycle inside all the channels associated with a given SST. Phase two of the IIDWBA algorithm is responsible for allocating bandwidth inside a given channel considering the actual bandwidth requests and minimum guaranteed bandwidth per CS per service cycle. At the end of a given bandwidth allocation cycle, the local information such as the total number of the heavily loaded CSs and associated MAC addresses along with the total excess bandwidth, total excess requested bandwidth and the generated service cycle will be passed to the third phase of the IIDWBA algorithm.

An algorithm and a flowchart, Figure 3, are developed for the phase two of the IIDWBA algorithm as follows.

\section{Intra-channel bandwidth allocations phase: algorithm and flowchart}

\section{Given:}

1) $T_{\text {cycle }}$ : Maximum time cycle which is the maximum time during which all the CSs get a chance to send data traffic and/or REPORT messages to a given SST.

2) $G$ : Guard time (gap) between two consecutive time-slots.

3) $R_{N}:$ Channel rate between the SST and CS.

4) $w_{i}$ : Allocated weight to a given $C S_{i}$ according to the SLA definitions where:

$$
{ }_{i=1}^{N} w_{i}=1
$$

in case of no SLA classification $w_{i}=\mathrm{W}=1 / \mathrm{N}$.

5) $\quad N$ : Number of the CSs associated with a given SST.

6) $H_{j}$ : Total number of the heavily loaded CSs on a given channel $j$.

7) $M A C{ }_{j}^{i}$ : MAC address associated with a given heavily loaded $C S_{i}$ on a given channel $j$.

8) $B_{\min _{j}}^{i}$ : Minimum guaranteed bandwidth for a given $C S_{i}$ on a given channel $j$, where: $j=1, \ldots, \alpha$ and $i=1, \ldots$, $N$. We require that:

$$
\underset{j=1}{j=\alpha} g \quad \underset{i=1}{i=N} B_{\min _{j}}^{i}<R_{N}
$$

9) $B_{r e q}{ }_{j}^{i}$ : Requested bandwidth from a given $C S_{i}$ on a given channel $j$.

10) $B_{g r a} a_{j}^{i}$ : Granted bandwidth to a given $C S_{i}$ on a given channel $j$.

11) $B_{\text {total_excess }_{j}}$ : Total excess bandwidth collected from a given channel $j$.

12) $B_{\text {avg_excess }}^{i}$ : Average granted excess bandwidth to a given heavily loaded $C S_{i}$ on a given channel $j$.

13) $B_{\text {total_excess_requested }}$ : Total extra requested bandwidth on a given channel $j$.
14) $C S_{t \text { duration }}^{i, n, j}$ : Allocated transmission duration to a given $C S_{i}$ during a given service cycle $n$ on a given channel $j$.

15) $C S_{t_{-} \text {start_time }}^{i, n, j}$ Allocated transmission start time to a given $C S_{i}$ during a given service cycle $n$ on a given channel $j$.

16) $R T T{ }_{j}^{i}$ : Round trip time for a given $C S_{i}$ on a given channel $j$.

\section{Define:}

1) $B_{\min _{j}}^{i}$ : Minimum guaranteed bandwidth for a given $C S_{i}$ on a given channel $j$. In this paper the same formula from [8] is employed in order to calculate $B_{\min _{j}}^{i}$ as follows.

$$
B_{\min _{j}}^{i}=\frac{\left(T_{c y c l e}-N \times G\right) x R_{N} \times w_{i}}{8}
$$

2) A given SST needs to generate a service cycle which includes the transmission start time and transmission duration for all the associated CSs at the end of each bandwidth allocation cycle as follows.

a. Transmission duration for a given $C S_{i}$ during a given service cycle $n$ on a given channel $j$ is calculated as follows where 64 bytes is required bytes to transmit the next MPCP Ext. REPORT message:

$$
C S_{t_{-} d u r a t i o n}^{i, n, j}=\left(\frac{B_{g r a}^{i}+64 \text { bytes }}{R_{N}}\right)+R T T_{j}^{i}
$$

b. Transmission start time for a given $C S_{i}$ during a given service cycle $n$ on a given channel $j$ is considered by taking into account the granted transmission duration to the previous CS $\left(C S_{i-1}\right)(6)$ as well as the guard time, $G$, between two consecutive allocated time-slots as follows.

$$
C S_{t \_ \text {start_time }}^{i, n, j}=C S_{t_{-} \text {duration }}^{i-1, n}+G
$$

In order to distinguish how the bandwidth is granted from a given SST to the associated CSs during a given service cycle $n$ on a given channel $j$, the Limited Bandwidth Allocation Scheme, which has been studied in [14] and [50], is discussed fist as follows.

In Limited Bandwidth Allocation Scheme, if the requested bandwidth from a given $\mathrm{CS}$ is less than the minimum guaranteed bandwidth, the requested bandwidth will be granted; otherwise, the minimum guaranteed bandwidth will be granted. This approach provides excess bandwidth remained from the CSs which requested bandwidth less than the minimum guaranteed bandwidth (lightly loaded CSs). One solution to employ the excess bandwidth is to distribute it fairly among those CSs which requested bandwidth more than the minimum guaranteed one (heavily loaded CSs) like the work proposed in [8]. However, the difference between the work in this paper and the work in [8] is that in this paper, the 
excess amount of bandwidth, which is remained from a given channel $j$ globally if it is not employed by the local CSs associated with it using phase two and phase three of the IIDWBA algorithm. The phase two of the IIWDBA algorithm, which happens inside all channels associated with a given SST, is firstly discussed, which is followed by phase three of the IIDWBA algorithm next.

First, all CSs are grouped into heavily loaded CSs and lightly loaded CSs. Lightly loaded CSs are those which are requested bandwidth less than the minimum guaranteed bandwidth and heavily loaded CSs are those which are requested bandwidth more than the minimum guaranteed bandwidth. Second, the SST starts granting bandwidth to the lightly loaded CSs and then heavily loaded CSs in sequence as follows.

Case I: The requested bandwidth from a given $C S_{i}$ on a given channel $j$ is less than or equal to the minimum guaranteed bandwidth, i.e.,

$$
B_{\text {req }}^{i} \leq B_{\min _{j}}^{i}
$$

In such a case, the requested bandwidth will be granted and the excess bandwidth will be collected, therefore:

$$
\begin{gathered}
B_{\text {gra }_{j}}^{i}=B_{\text {req }}^{i} \\
B_{\text {total_excess }_{j}}={ }_{i=1}^{i=N} B_{\text {min }_{j}^{i}}^{i}-B_{\text {req }}^{i}
\end{gathered}
$$

Case II: The requested bandwidth from a given $C S_{i}$ on a given channel $j$ is more than the minimum guaranteed bandwidth, i.e.,

$$
B_{r e q_{j}}^{i}>B_{\min _{j}}^{i}
$$

Therefore:

$$
B_{\text {total_excess_requested }_{j}}=\quad{ }_{i=1}^{i=N} B_{\text {req }}^{i}{ }_{j}^{i}-B_{\min _{j}}^{i}
$$

In such a case, the minimum guaranteed bandwidth and average of the total excess bandwidth (13) will be considered to be allocated, i.e.,

$$
B_{\text {avg_excess }_{j}}^{i}=B_{\text {total_excess }_{j}} / H_{j}
$$

which brings following two cases:

Case II. A: The requested bandwidth from a given heavily loaded $C S_{i}$ on a given channel $j$, is less than or equal to sum of the minimum guaranteed bandwidth and average of total excess bandwidth, i.e.,

$$
B_{\text {req }}^{i} \leq B_{\text {min }_{j}^{i}}^{i}+B_{\text {avg_excess }_{j}^{i}}^{i}
$$

In such a case, $B_{r e q}{ }_{j}^{i}$ will be granted and the excess will be collected. As a result, the correspondent CS will not be considered as lightly loaded CS anymore, therefore:

$$
\begin{gathered}
B_{g r a_{j}^{i}}^{i}=B_{r e q}^{i} \\
H_{j}{ }^{--} \\
B_{\text {total_excess }_{j}}+={ }_{i=1}^{i=N} B_{\text {min }_{j}}^{i}+B_{\text {avg_excess }_{j}^{i}}^{i}-B_{\text {req }}^{i} \\
B_{\text {total_excess_requested }_{j}}-={ }_{i=1}^{i=N} B_{\text {req }}^{i}{ }_{j}^{i}-B_{\text {min }_{j}}^{i}
\end{gathered}
$$

Case II. B: The requested bandwidth from a given heavily loaded $C S_{i}$ on a given channel $j$, is more than the sum of the minimum guaranteed bandwidth and average of the total excess bandwidth, i.e.,

$$
B_{\text {req }}^{i}>B_{\text {min }_{j}}^{i}+B_{\text {avg_excess }_{j}}^{i}
$$

In such a case, $\left(B_{\min _{j}}^{i}+B_{\text {avg_excess }}^{i}\right)$ will be granted, and the corresponding CS will be remained heavily loaded, therefore:

$$
\begin{array}{r}
B_{g_{g r a}{ }_{j}}^{i}=B_{\text {min }_{j}}^{i}+B_{\text {avg_excess }_{j}}^{i} \\
B_{\text {total_excess_requested }_{j}}-=B_{\text {avg_excess }_{j}}^{i}
\end{array}
$$

In this stage, the local scheduler, which is associated with

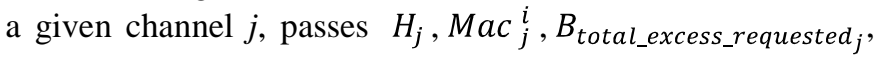
$B_{\text {total_excess }_{j}}$ and the latest schedule service cycle to the global scheduler on a given SST in order to execute phase three of the IIDWBA algorithm termed Inter-channel bandwidth allocations phase, which will be discussed later. The associated flowchart for the phase two of the IIDWBA algorithm is represented in Figure 3.

As it is depicted in Figure 3, at the first step, each CS will be identified either as a lightly loaded or a heavily loaded CS. The lightly loaded CSs will be granted the requested bandwidth, and the excess bandwidth will be collected from them at the same time. Then the bandwidth will be allocated to the heavily loaded CSs such that it will be no more than the minimum guaranteed bandwidth plus the average amount of the excess bandwidth which is collected from the lightly loaded CSs.

After allocating bandwidth to all CSs, including the lightly loaded and heavily loaded ones, the local information such as the total number of the local heavily loaded CS and associated MAC addresses as well as the individual excess requested bandwidth along with the total excess bandwidth, total excess requested bandwidth and current scheduled service cycle will be passed from the phase two to phase three of the IIDWBA algorithm. 


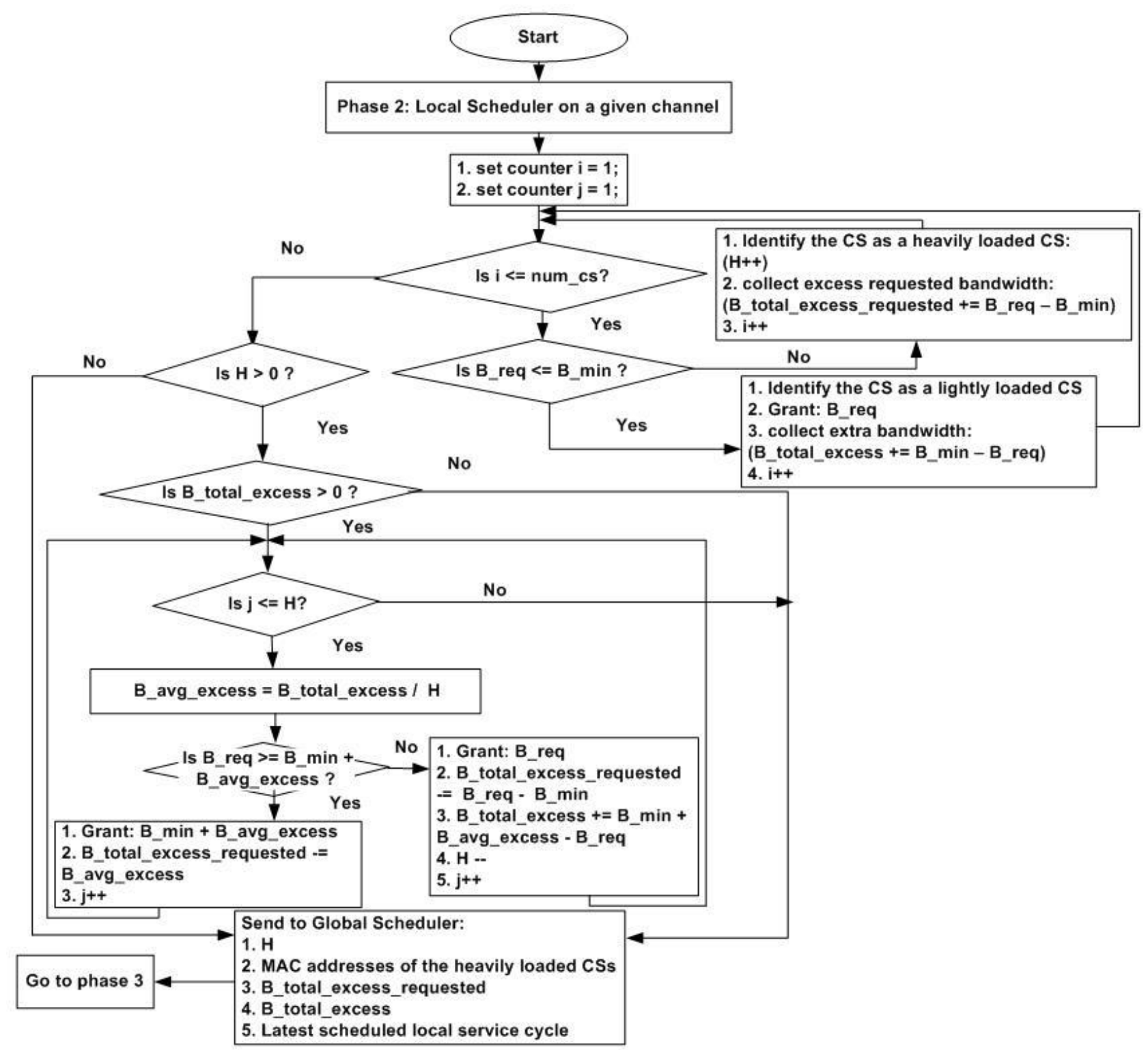

Figure 3 Flowchart for Intra-channel bandwidth allocations phase of the IIDWBA algorithm

2. Summary of the general function for Intra-channel bandwidth allocations phase (phase two) of the IIDWBA algorithm:

1) Allocating bandwidth to local CSs on a given channel $j$.

2) Scheduling the local service cycle.

3) Capturing the total number of the heavily loaded CSs with the associated MAC addresses, total local excess bandwidth and total local excess requested bandwidth.

4) Sending the scheduled service cycle from objective 2 along with the captured information from objective 3 to the Global scheduler.

The next section provides a detailed discussion for the phase three of the IIDWBA algorithm as follows.

\section{Inter-channel bandwidth allocations phase}

Phase three of the IIDWBA algorithm, which is termed Inter-channel bandwidth allocations phase, runs immediately on a given SST when the phase two execution have been finished over all the channels. It includes three real-time stages of Collect, Schedule and Distribute as follows.

During the Collect stage, phase three of the IIDWBA algorithm starts collecting the local information from each single channel, including the number or the local heavily loaded CSs with associated MAC addresses, total excess bandwidth and total excess requested bandwidth along with the latest scheduled service cycle.

During the Schedule stage, based on the number of the total heavily loaded CSs on all the channels (globally heavily 
loaded CSs) and global excess requested bandwidth as well as the global excess available bandwidth, phase three of the IIDWBA algorithm schedules the global excess bandwidth among all the globally heavily loaded CSs.

During the Distribute stage, the globally scheduled excess bandwidth will be distributed among the globally heavily loaded CSs inside the associated service cycle and immediately broadcast to all the channels.

Generally speaking, the main concern in phase three of the IIDWBA algorithm is to find and collect the real-time excess amount of bandwidth from all the available channels during each service cycle and spend (schedule and distribute) them immediately on those CSs which are identified as globally heavily loaded in the latest service cycle.

An algorithm and a flowchart, Figure 4, are developed for the phase three of the IIDWBA algorithm as follows.

\section{Inter-channel bandwidth allocations phase: algorithm and flowchart}

\section{Given:}

1) $M A C_{j}:$ All MAC addresses associated with the heavily loaded CSs on a given channel $j$.

2) $T_{j}:$ Latest scheduled service cycle associated with a given channel $j$.

3) $T_{\text {last_t_start time }}^{j}$ : Last transmission start time allocated inside a latest service cycle associated with a given channel $j$.

4) $T_{\text {last_t_duration }}^{j}$ : Last transmission duration allocated inside a latest service cycle associated with a given channel $j$.

\section{Define:}

1) $H_{\text {global }}$ : Total number of the heavily loaded CSs across all the channels:

$$
H_{\text {global }}=\underset{j=1}{j=\alpha} H_{j}
$$

2) $B_{\text {excess_global }}$ : Total excess bandwidth collected from all the channels:

$$
B_{\text {excess_global }}=\quad \underset{j=1}{j=\alpha} B_{\text {total_excess }_{j}}
$$

3) $B_{\text {avg_excess_global }}$ : Average global excess bandwidth available for a given globally heavily loaded $C S_{i}$ :

$$
B_{\text {avg_excess_global }}=B_{\text {excess_global }} / H_{\text {global }}
$$

In this stage, the global scheduler received required information, including: number of the heavily loaded CSs, $H_{j}$, and associated MAC addresses, $M a c_{j}^{i}$, along with the total excess bandwidth, $B_{\text {total_excess }}$, total excess requested bandwidth, $B_{\text {total_excess_requested }}$, as well as the last scheduled service cycle from each channel.

The phase three of the IIDWBA algorithm works through the following cases.

Case I: There is no heavily loaded CS across all the channels.

$$
H_{\text {global }}=0
$$

Case I brings two other cases as follows.

Case I. A: All the CSs across a given channel $j: j=1, \ldots, \alpha$ are lightly loaded, which means they are all requested bandwidth equal to or less than the minimum guaranteed bandwidth.

Case I. B: Some of the CSs across a given channel $j: j=$ $1, \ldots, \alpha$ are heavily loaded and some are lightly loaded. However, the local heavily loaded CSs are satisfied with the excess bandwidth collected from the local lightly loaded CSs. Therefore, the local heavily loaded CSs are not globally heavily loaded.

In such cases, Case I. A and Case I. B, the global scheduler passes all the received service cycles from all the channels to the Broadcaster which will then broadcast to all the channels immediately.

Case II: There are heavily loaded CSs across channels but there is no global excess bandwidth.

$$
\begin{aligned}
& H_{\text {global }}>0 \\
& B_{\text {excess_global }}=0
\end{aligned}
$$

Case II brings two other cases as follow.

Case II. A: All the CSs across a given channel $j: j=1, \ldots, \alpha$ are heavily loaded. Therefore, there is no local excess bandwidth available from a given channel $j: j=1, \ldots, \alpha$ to satisfy local demands for the excess requested bandwidth.

Case II. B: Some of the CSs across a given channel $j$ : $j=$ $1, \ldots, \alpha$ are heavily loaded and some are lightly loaded. However, the local heavily loaded CSs are not satisfied with the excess bandwidth collected from the local lightly loaded CSs. Therefore, some of or all of the local heavily loaded CSs are also globally heavily loaded.

In such cases, Case II. A and Case II. B, the global scheduler passes all the received service cycles from all the channels to the Broadcaster which will then broadcast to all the channels immediately.

Case III: There are heavily loaded CSs across all the channels and the global excess bandwidth is available.

$$
H_{\text {global }}>0
$$




$$
B_{\text {excess_global }}>0
$$

In such a case, the global scheduler allocates the global excess bandwidth among globally heavily loaded CSs scattered across all channels.

In first step, average global excess bandwidth (24) will be calculated by the global scheduler for all heavily loaded CSs which may not be located in a same channel.

In second step, the global scheduler compares the average global excess bandwidth with total excess requested bandwidth from each channel, which brings the following two cases:

Case III. A: The average global excess bandwidth calculated for all heavily loaded CSs on a given channel $j: j=1, \ldots, \alpha$, is more than or equal to the total excess requested bandwidth from associated channel:

$B_{\text {total_excess_requested }_{j}} \leq B_{\text {avg_excess_global }_{i}} * H_{j}$

In such a case, $B_{\text {total_excess_requested }}$ will be granted.

Case III. B: The average global excess bandwidth calculated for all heavily loaded CSs on a given channel $j: j=1, \ldots, \alpha$, is less than the total excess requested bandwidth from the associated channel:

$B_{\text {total_excess_requested }_{j}}>B_{\text {avg_excess_global }_{i}} * H_{j}$

In such a case, $B_{\text {avg_excess_global }} * H_{j}$ will be granted.

The granted bandwidth then will be scheduled inside the appropriate service cycle, $T_{j}$, by global scheduler taking into account: $T_{\text {last_t_start_time }}^{j}$ and $T_{\text {last_t_duration }}^{j}$ as well as $M A C_{j}$.

Then, the global scheduler passes all the service cycles to the Broadcaster which will broadcast to all channels immediately.

The associated flowchart for the phase three of the IIDWBA algorithm is represented in Figure 4.

As it is shown in Figure 4, phase three of the IIDWBA algorithm first receives the local information from all the channels associated with a given SST which includes the total number of the heavily loaded CSs, associated MAC addressee and individual excess requested bandwidth, total excess bandwidth, total excess requested bandwidth and the latest scheduled service cycles. Then it distributes the total excess bandwidth, which is collected from all the channels, among all the globally heavily loaded CSs. The distributed global excess bandwidth will be scheduled inside the appropriate service cycles associated with the lightly loaded channels and will be passed to the Broadcaster in order to immediately broadcast to all CSs scattered over all channels.

\section{Summary of the general function for Inter-channel bandwidth allocations phase (phase three) of the IIDWBA algorithm:}

1) Receiving the total number of the heavily loaded CSs with associated MAC addresses, total local excess bandwidth and total local excess requested bandwidth from all the channels associated to a given SST.

2) Receiving the latest scheduled service cycle from all the channels.

3) Identifying the lightly loaded and heavily loaded channels.

4) Calculating the total number of the heavily loaded CSs across all the heavily loaded channels (globally heavily loaded CSs).

5) Calculating the total excess bandwidth across all the lightly loaded channels (global excess bandwidth).

6) Calculating the average granted excess bandwidth from each lightly loaded channel to a given globally heavily loaded CS.

7) Allocating the global excess bandwidth to the globally heavily loaded CSs according to the actual need.

8) Scheduling and embedding the global allocated excess bandwidth inside service cycle of each lightly loaded channel.

Sending all the service cycles for all the channels to broadcaster.

The next Section explains the full IIDWBA operations through an example in order to help understanding its functionality during each phase.

\section{An example for three phases of the IIDWBA algorithm operations}

As it is depicted in Figure 5, on a given SST, e.g. the OLT in the $\mathrm{CO}$, each channel is associated with a scheduler termed a local scheduler which is responsible for allocating the bandwidth from the local channel among all the CSs assigned to it.

A given local scheduler also deals with the master scheduler termed a global scheduler on a given SST. While a local scheduler is only in charge of allocating bandwidth among its associated CSs, a given global scheduler deals with all the channels on a given SST and responsible for allocating the global excess bandwidth from all the channels among the globally heavily loaded CSs which may exist over different channels. Generally speaking a given local scheduler on a given SST is responsible for the following tasks: 1) Identifying the local lightly/heavily loaded CSs 2) Granting bandwidth to the lightly loaded CSs 3) Collecting bandwidth from the lightly loaded CSs 4) Distributing the local excess bandwidth among local heavily loaded CSs 5) Passing the following information to the global scheduler: the total number of the heavily loaded CSs along with the associated MAC addresses and individual excess requested bandwidth, the total excess bandwidth, the total requested excess bandwidth along with the latest scheduled service cycle. This information is termed as clarification data.

When the global scheduler receives the clarification data from all the associated channels on a given SST, it first starts counting the number of the heavily loaded CSs, which may be 


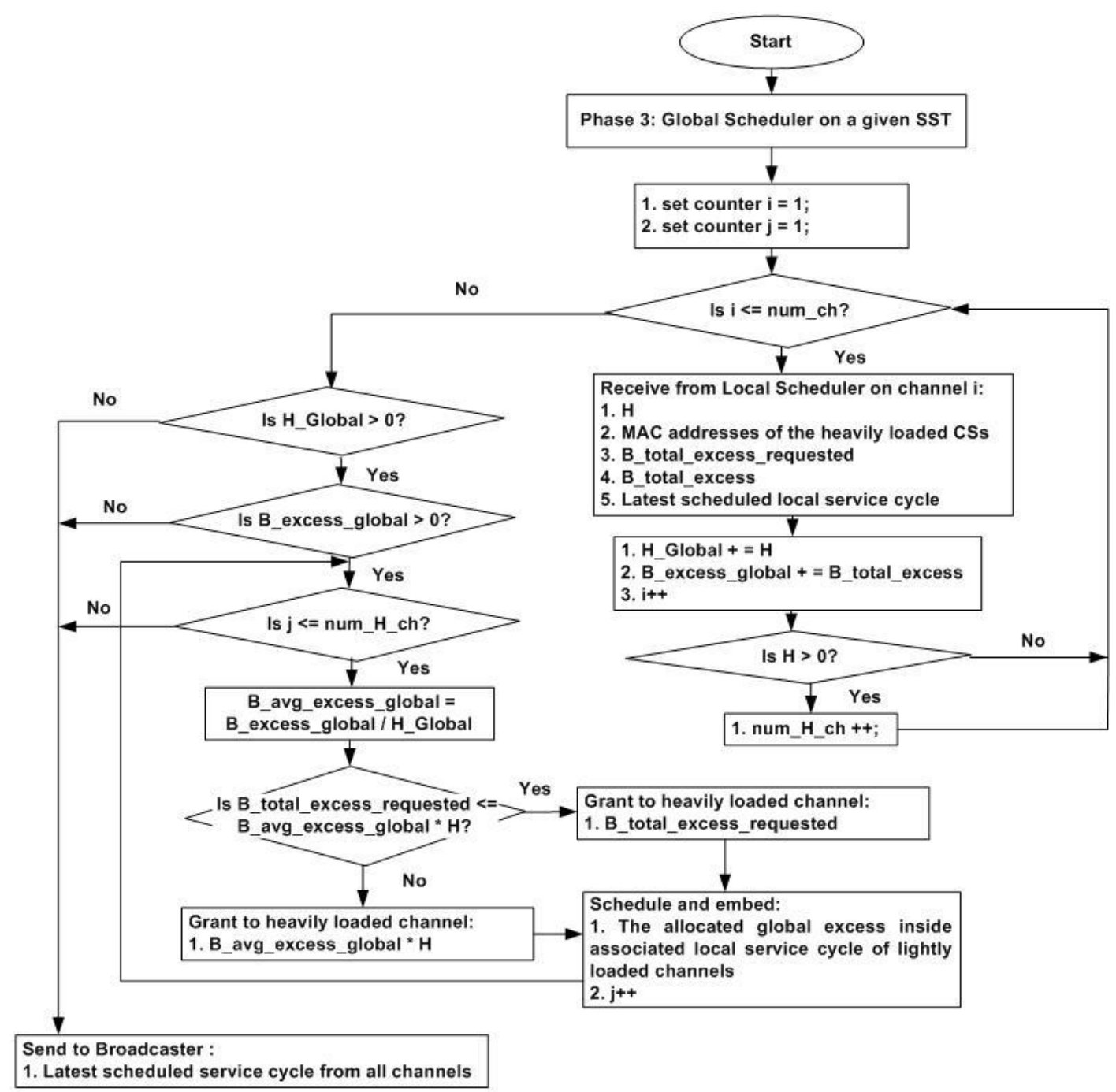

Figure 4 Flowchart for Inter-channel bandwidth allocations of the IIDWBA algorithm

scattered across different channels, along with the total excess bandwidth and total excess requested bandwidth collected from all the channels. A given globally heavily loaded CS will be allocated with the global excess bandwidth in such a way that it will be guaranteed the average portion of the total excess bandwidth collected from all the channels and will not receive bandwidth more than its requirements. The allocated global excess bandwidth to the globally heavily loaded CSs will be scheduled and embedded inside the service cycle associated with the lightly loaded channels. After finishing the service cycle modifications, all the service cycles will be passed to the Broadcaster which will be then broadcasted to all channels associated with a given SST, Figure 5.

In order to display the output of the IIDWBA algorithm after execution of all three phases, a scenario of a given SST with four associated channels (channel_l to channel_4) in which each channel is assigned to four CSs, is made. Two traffic patterns termed as first traffic pattern and second traffic pattern are then identified as follow.
In the first traffic pattern, the load on channel_l is gradually increased while the load on other channels (channel_2 to channel_4) are fixed. In this case, in a given service cycle $T_{1}$, the SST identifies channel_l as a heavily loaded channel and three other channels (channel_2 to channel_4) as lightly loaded channels. Thus, IIDWBA algorithm starts collecting the available excess bandwidth from (channel_2 to channel_4) in order to distribute them among CSs associated with channel_l and respond to their high bandwidth requests. A given modified service cycle which will be passed to the Broadcaster at the end of the IIDWBA algorithm executions includes the allocated time slots for the local CSs as well as the extra allocated time-slots which are allocated to the globally heavily loaded CSs associated with channel_1. When a modified service cycle broadcast to the CSs, a given heavily loaded CS, which is locally registered to channel_l in above example, will find itself with multiple time-slot assignments on the local registered channel, channel_1, as well as three other lightly loaded channels, channel_2 to channel_4, Figure 6. 


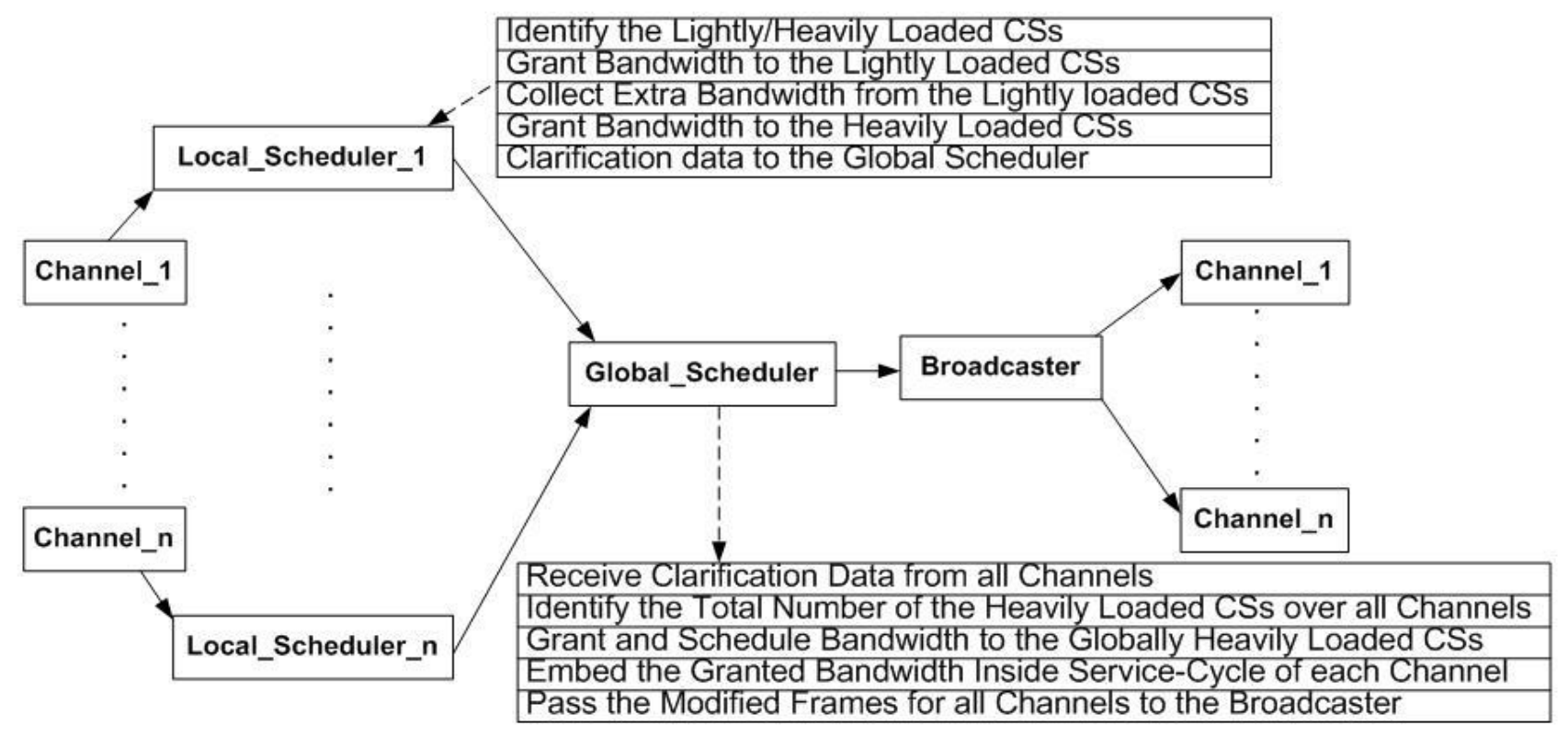

Figure 5 Execution of the IIDWBA algorithm inside a given SST

In the second traffic pattern, the load on all channels, channel_1 to channel_4, is simultaneously increased in order to display how IIDBA algorithm responds when no global excess bandwidth is identified across all the channels associated to a given SST. In this case, in a given service cycle $\mathrm{T}_{2}$, the global scheduler, which is implemented inside the SST, identifies that there is no local excess bandwidth on all its associated channels. Therefore, it will broadcast all the received service cycles from its associated channels to the broadcaster immediately, Figure 7.

The required messages, which need to be passed during the execution of the IIDWBA over a given service cycle in order to provide the successful execution of the proposed algorithm and meet the second objective of this thesis, are shown in Figure 8.

The next section evaluates the performance of the IIDWBA algorithm through different simulated scenarios and traffic pattern configurations as follows.

\section{IMPLEMENTATIONS}

In this Section, the performance of the proposed IIDWBA algorithm over the converged network is evaluated by conducting a simulated scenario using OPNET Modeler [9]. A converged scenario is implemented where a network of a single OLT, 16 ONUs, and 16 BSs per ONU are simulated, Figure 9. In the simulated scenario, the IIDWBA algorithm is implemented over the OLT and all ONUs. The components of the converged scenario are as follows.

A single OLT in CO, which supports four channels $\left(w_{1}, \ldots, w_{4}\right)$ and is related to 16 ONUs, a 1:4 sized Arrayed Waveguide Grating (AWG) with a co-located amplifier and four 1:16 sized TDM Splitters. AWG and TDM Splitters are seated between the OLT and ONUs, respectively. A given $\mathrm{ONU}$ is also assigned to $16 \mathrm{BSs}$ and supports the same four channels, $\left(w_{1}, \ldots, w_{4}\right)$.

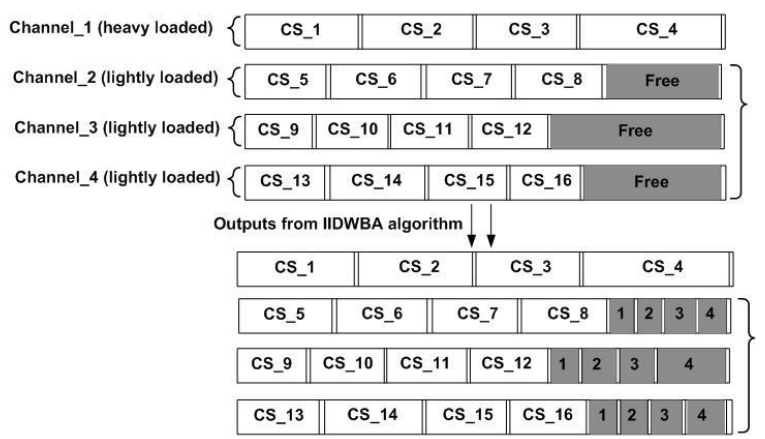

Figure 6 Output from IIDWBA algorithm for a given service cycle $T_{1}$ (traffic pattern one)

\begin{tabular}{|c|c|c|c|c|}
\hline Channel_1 (heavy loaded) & CS_1 & CS_2 & Cs_3 & Cs_4 \\
\hline Channel_2 (heavy loaded) & CS_5 & CS_6 & CS_7 & CS_8 \\
\hline Channel_3 (heavy loaded) & CS_9 & CS_10 & Cs_11 & CS_12 \\
\hline Channel_4 (heavy loaded) \{ & CS_13 & CS_14 & CS_15 & CS_16 \\
\hline \multicolumn{5}{|c|}{ Outputs from IIDWBA algorithm } \\
\hline & CS_1 & Cs_2 & Cs_3 & CS_4 \\
\hline & CS_5 & CS_6 & Cs_7 & CS_8 \\
\hline & CS_9 & CS_10 & Cs_11 & CS_12 \\
\hline & CS_13 & CS_14 & CS_15 & CS_16 \\
\hline
\end{tabular}

Figure 7 Output from IIDWBA algorithm for a given service cycle $T_{2}$ (traffic pattern two) 


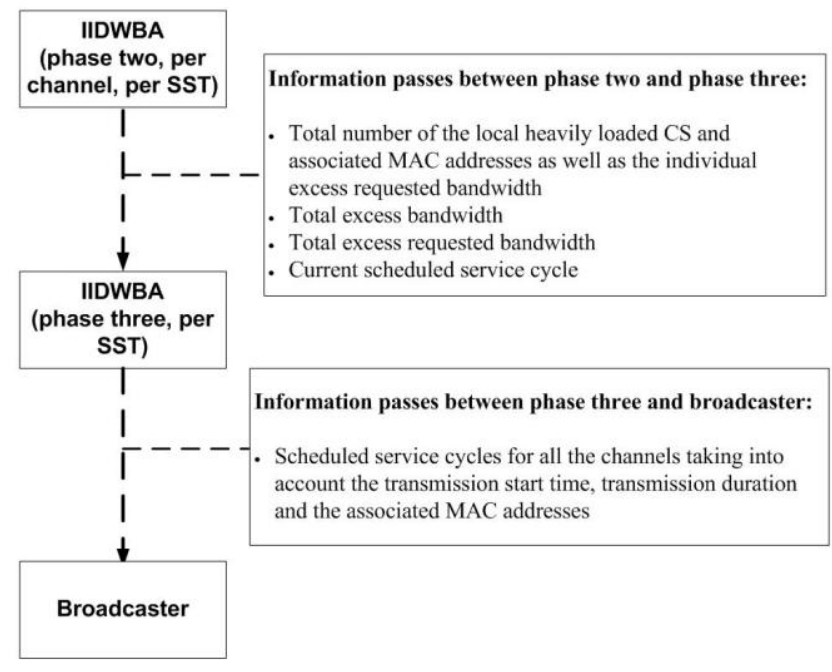

Figure 8 Information passes over the execution of the IIDWBA algorithm

A 1:4 sized AWG with a co-located amplifier and four 1:16 sized TDM Splitters are also located between a given ONU and BSs, respectively. Each BS is also associated with 10 wireless SSs which will be increased to 100 SSs in stage by 10 in order to evaluate the performance of the proposed IIDWBA algorithm under the different load values. Therefore, the total number of the ONUs, BSs and SSs are 16, $256(16 \mathrm{ONU} \times 16 \mathrm{BS})$ and 2560 to $25600(256 \mathrm{BS} \times 10 \mathrm{SS}$ to $256 \mathrm{BS} \times 100 \mathrm{SS})$, respectively in the whole converged system. A given BS also supports the same four channels $\left(w_{1}, \ldots, w_{4}\right)$ for the upstream transmissions towards the associated ONU. The total distance supported by the converged simulated model from a given wireless subscriber station at front-end customer premises up to the OLT in CO is $\sim 300 \mathrm{~km}$. Simulation parameters, Table.2, are employed for the experiments over the converged scenario. Based on the Table.2 specifications, traffic flows with: burst pattern, different ON/OFF state time, constant packet size, exponentially-distributed packet inter-arrival time and uneven distributions across BSs are employed over the converged simulated scenario.

AWG is a successful device in WDM-PON industry, which has been used in many long-reach WDM systems. It is capable of routing each wavelength to a unique out port, separating the multiple wavelengths from each other as well as combining and sorting different channels. Moreover, it can be a multiplexor as well as a de-multiplexor at the same time based on the cyclic wavelength property features. $\mathrm{N} \times \mathrm{N}$ AWG is usually called $A W G$ router as it can realize $\mathrm{N} \times \mathrm{N}$ optical wavelength router using only a single component [40]. When the free-spectral range of $\mathrm{N} \times \mathrm{N} A W G$ is $\mathrm{N}$ times, it is capable of processing $\mathrm{N} \times \mathrm{N}$ optical interconnection in a nonblocking way because of the cyclic property of the output wavelengths [41]. AWG can be pumped in with the different number of wavelengths with same/different bit rates each supplied for a single out port. For instance, if $\mathrm{N}$ wavelengths pumped into a given $\mathrm{N} \times \mathrm{N}$ AWG all with the same capacity of $1 \mathrm{~Gb} / \mathrm{s}$, each out port will ideally pick up one of the input wavelengths with the equal capacity of $1 \mathrm{~Gb} / \mathrm{s}$. In this paper, 4 $\times 4$ AWGs, which are pumped in with 4 wavelengths each with equal capacity of $1 \mathrm{~Gb} / \mathrm{s}$, are capable of providing equal $1 \mathrm{~Gb} / \mathrm{s}$ capacity on each out port. The provided capacity will be then forwarded to a single 1: 16 passive splitter/combiner and will be shared among associated CSs (ONUs/BSs), Figure 9.

1: $\mathrm{N}$ passive splitter/combiner is one of the key components in FTTX world, which includes the typical parameters such as: input cable length, output cable length, splitting ratio, connector types and working wavelengths [42]. 1: $\mathrm{N}$ passive splitter/combiner is a plain power splitter in which the input power will be equally divided based upon the number of the available/supported/active out ports. For instance, if a plain 1:16 passive splitter/combiner is pumped in with the power capacity of $1 \mathrm{~Gb} / \mathrm{s}$, each out port will identically receive about $62.5 \mathrm{Mb} / \mathrm{s}$ bit rates $(1 \mathrm{~Gb} / \mathrm{s}$ divided by 16). In this paper, $\mathrm{N}$ : 16 passive splitter/combiners are employed in which each input capacity will be equally divided among 16 out ports for 16 CSs. For instance, when the number of the available wavelengths (with $1 \mathrm{~Gb} / \mathrm{s}$ capacity each) on the OLT is four, the available capacity for each ONU will be $4 \times 62.5 \mathrm{Mb} / \mathrm{s}$.

Based upon widely used configurations for the converged scenario and traditional TDM-based PON [37], [14], the simulated scenario is implemented as follows.

The buffer sizes inside the ONUs and BSs are set to finite 10 Mbytes and the maximum cycle time is considered as 2 ms. Maximum cycle time is the maximum time that a given $\mathrm{CS}$ (ONU/BS) gets a chance for the upstream data transmissions towards associated SST (OLT/ONU), correspondingly. Fixed $192 \mu \mathrm{s}$ is considered as RTT delay for each CS (ONU/BS) in every service cycle. Moreover, 100 $\mathrm{Mb} / \mathrm{s}$ and $1 \mathrm{~Gb} / \mathrm{s}$ are the upstream data rates between a given $\mathrm{BS}$ and the associated ONU as well as a given ONU and the OLT, both correspondingly. Guard time, which is separating two consecutive time slots, needs to be considered in order to support several factors such as laser switching delay, ranging inaccuracy, and clock-recovery preamble. The lower bound for the guard time is laser switching time, which is about 10ns, as optical light sources need to be turned off completely when they are not transmitting. Therefore, guard time of sub$\mu \mathrm{s}$ is reasonable [49]. In this paper, a fixed guard time of $5 \mu \mathrm{s}$ is considered for the light sources on ONUs and BSs. Moreover, MPCP Ext. protocol is employed in order to support all the communications among the components of the converged scenario.

At the network setup stage, phase one of the IIDWBA algorithm (Initialisation phase), which is implemented on all the SSTs including the OLT in CO and all the ONUs, associates four ONUs as well as four BSs to each channel. Phase two of the IIDWBA algorithm (Intra-channel bandwidth allocations phase) then starts sharing the available channels among the CSs associated with all the SSTs (each channel on OLT will be shared among four ONUs and each channel on a given ONU will be shared among four BSs). When the traffic starts building up on the SSs over the simulated scenario, phase three of the IIDWBA algorithm 


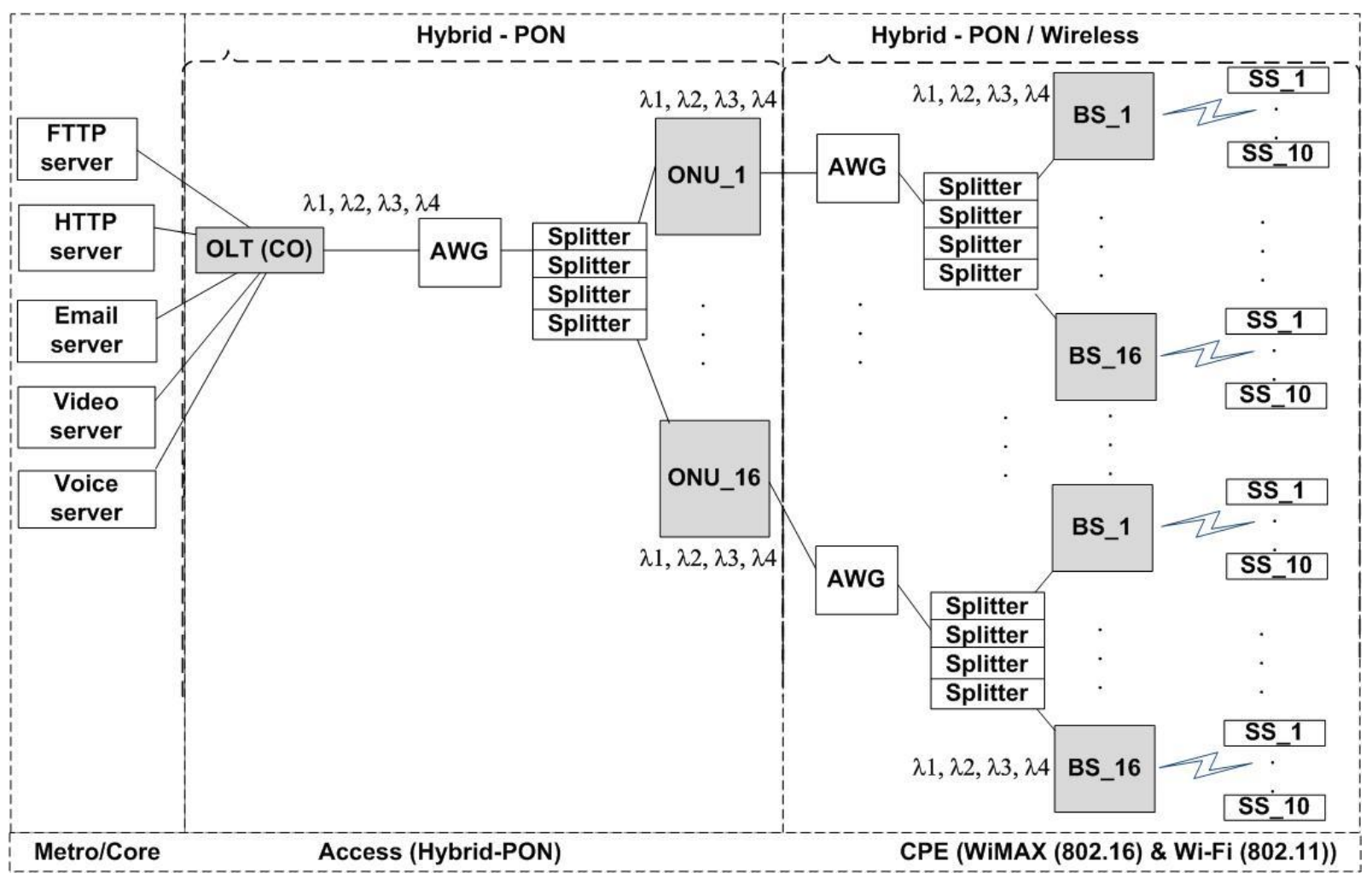

Figure 9 Network topology for evaluating the proposed IIDWBA algorithm

(Inter-channel bandwidth allocations phase) will be executed independently on all the SSTs (the OLT and ONUs). On a given SST, it collects the free time-slots from all the lightly loaded channels and then passes them to the global scheduler. The global scheduler then schedules and distributes the free collected time-slots among the heavily loaded CSs scattered all over the heavily loaded channels. Finally, the MPCP Ext. will be employed in order to carry the allocation decisions to the CSs associated with a given SST. The performance of the IIDWBA algorithm is considered when the execution of all three phases is finished. As the work in this paper is mainly focused on phase three of the IIDWBA algorithm, while the other two phases act as building blocks, the phase three results are only discussed within this paper.

Table.2 SIMULATION PARAMETERS

\begin{tabular}{|l|l|}
\hline Traffic pattern & Burst (uneven across SSs) \\
\hline ON state time $(\mathrm{sec})$ & $20 \%$ of simulation time \\
\hline OFF state time $(\mathrm{sec})$ & $80 \%$ of simulation time \\
\hline Traffic start time & even across all SSs \\
\hline Traffic stop time & Never \\
\hline Packet size & 500 bytes $($ constant) \\
\hline $\begin{array}{l}\text { Number of channels supported on } \\
\text { OLT, ONUs and BSs for upstream } \\
\text { transmissions }\end{array}$ & 4 channels $\left(w_{1}, \ldots, w_{4}\right)$ \\
\hline Number of SSs pr BS & 10 to $100 \mathrm{BY} 10$ \\
\hline Traffic class & Best Effort $(\mathrm{BE})$ \\
\hline Simulation time, seed & 30 sec, 128 \\
\hline value per static, update interval & 1600,300000 \\
\hline
\end{tabular}

In order to evaluate the phase three of the IIDWBA algorithm, an uneven traffic pattern is considered across all the channels $\left(w_{1}, \ldots, w_{4}\right)$ on a given SST where lightly loaded as well as heavily loaded CSs are always available during the simulation run time. The lightly loaded channels are those channels allocated to the CSs which requested bandwidth less than or equal to the minimum guaranteed bandwidth. The heavily loaded channels are those channels allocated to the CSs which requested bandwidth more than the minimum guaranteed bandwidth. The simulation experiments are started with the following two traffic patterns configurations termed first traffic pattern and second traffic pattern as follows, Table.3.

In the first traffic pattern, the load on $w_{1}$ is gradually increased from $10 \mathrm{SSs}$ to $100 \mathrm{SSs}$ per BS while the number of the SSs on other channels $\left(w_{2}, \ldots, w_{4}\right)$ are fixed, up to $10 \mathrm{SSs}$ per BS, in order to distinguish how CSs on $w_{1}$ benefit from the available free bandwidth on the other channels $\left(w_{2}, \ldots, w_{4}\right)$ when the traffic builds up. In the second traffic pattern, the load on all channels, $w_{1}, \ldots, w_{4}$, is simultaneously increased by gradually raising the number of the SSs per BS from 10 to 100 in order to distinguish the performance of the CSs associated with $w_{1}$ in comparison with the results captured from the first traffic pattern.

Due to the simplicity of the downstream transmissions, which resemble the Ethernet broadcast behaviour; the experiments towards the upstream directions (to the OLT) are only considered in this paper. 
Table.3 TRAFFIC PATTERN CONFIGURATIONS

\begin{tabular}{|l|l|l|}
\hline $\begin{array}{l}\text { Traffic } \\
\text { pattern }\end{array}$ & Number of SSs on $\boldsymbol{w}_{\mathbf{1}}$ & $\begin{array}{l}\text { Number of SSs on } \\
\boldsymbol{w}_{\mathbf{2}}, \ldots, \boldsymbol{w}_{\mathbf{4}}\end{array}$ \\
\hline $\begin{array}{l}\text { First } \\
\text { traffic } \\
\text { pattern }\end{array}$ & $\begin{array}{l}\text { Gradually increased from } \\
\text { 10 SSs to 100 SSs per BS } \\
\text { (total of 160 to 1600 SSs } \\
\text { per ONU) }\end{array}$ & $\begin{array}{l}\text { Fixed 10 SSs per BS (total of } \\
160 \text { SSs to 1600 SSs per ONU) }\end{array}$ \\
\hline $\begin{array}{l}\text { Second } \\
\text { traffic } \\
\text { pattern }\end{array}$ & $\begin{array}{l}\text { Gradually increased from } \\
\text { 10 SSs to 100 SSs per BS } \\
\text { (total of 160 to 1600 SSs } \\
\text { per ONU) }\end{array}$ & $\begin{array}{l}\text { Gradually increased from 10 100 SSs per BS (total of } \\
160 \text { to 1600 SSs per ONU) }\end{array}$ \\
\hline
\end{tabular}

The same three phases of the proposed IIDWBA algorithm with the same configurations is implemented and employed between a given ONU and the associated BSs.

\section{CAPTURED RESUlts}

In this Section, the captured results after employing the IIDWBA algorithm are compared with the work presented in [8]. In [8], the authors proposed an algorithm in which the excess bandwidth remained from the local lightly loaded CSs will be collected and then distributed between the local heavily loaded CSs. However, the IIDWBA algorithm is able to identify, collect and then distribute the excess amount of bandwidth, which is remained from a given channel, globally if it is not employed by the local associated CSs using phase two and phase three. The captured results are as follow.

As Figure 10 reveals, when we employed the first traffic pattern, the proposed IIDWBA algorithm is successful in decreasing the average queuing delay to almost $14 \%$ for the ONUs associated with $\mathrm{w}_{1}$, when it is compared to the scenario without applying IIDWBA algorithm [8]. It is because when the load starts increasing on channel $\mathrm{w}_{1}$, the IIDWBA algorithm starts looking for the excess bandwidth on neighbour channels, $\mathrm{w}_{2}, \ldots, \mathrm{w}_{4}$, which will be collected and distributed among the ONUs associated with $\mathrm{w}_{1}$. The average queuing delay for the ONUs associated with $\mathrm{w}_{2}, \ldots, \mathrm{w}_{4}$ is also captured with and without applying IIDWBA algorithm when the first traffic pattern is employed, Figure 11. As Figure 11 depicts, the IIDWBA algorithm has almost zero negative effect on the performance of the ONUs associated with $\mathrm{w}_{2}, \ldots, \mathrm{w}_{4}$ as the allocated bandwidth form them is the unutilised local excess bandwidth. In Figure 12, the average extra requested bandwidth from the ONUs associated with the $\mathrm{w}_{1}$ is presented with and without the IIDWBA algorithm after employing the first traffic pattern. As Figure 12 reveals, the IIDWBA algorithm is capable of keeping the average extra requested bits from the ONUs associated with $\mathrm{w}_{1}$ under the minimum guaranteed bandwidth by allocating the available excess bandwidth from the other channels, $\mathrm{w}_{2}, \ldots, \mathrm{w}_{4}$, to them during each service cycle. In the second traffic pattern, The load on all channels is gradually increased by raising the total number of the SSs connected per BS from 10 to 100 in order to distinguish how the increased load on three channel, $\mathrm{w}_{2}, \ldots, \mathrm{w}_{4}$, will affect the performance of the ONUs over $\mathrm{w}_{1}$. As Figure 13 reveals, when the number of the SSs connected per BS on all the channel reaches to 60 , the queuing delay inside the ONUs associated with $w_{1}$ starts increasing constantly and achieved to almost $0.03 \mathrm{sec}$ when it gets to $100 \mathrm{SSs}$ per BS. The reason behind this degradation is that when the load on three channels, $w_{2}, \ldots, w_{4}$, is increased gradually the IIDWBA algorithm cannot find as much excess bandwidth for the ONUs associated with $w_{1}$ when it is compared to the first traffic pattern where the load on three channels is almost fixed. However, the queuing delay for the ONUs over $w_{1}$ under the second traffic pattern is still much lower than the scenario when the IIDWBA did not employ, Figure 10. In Figure 14, the average extra requested bandwidth (bits) over $w_{1}$ is also captured when the second traffic pattern is employed and then compared with the captured results from the first traffic pattern. As Figure 14 reveals, when the number of the SSs connected per BS is gradually increased from 50 to $100 \mathrm{SSs}$ on all channels, the average extra requested bits over $\mathrm{w}_{1}$ start raising almost twice more than the first traffic pattern scenario. The reason for such a result is

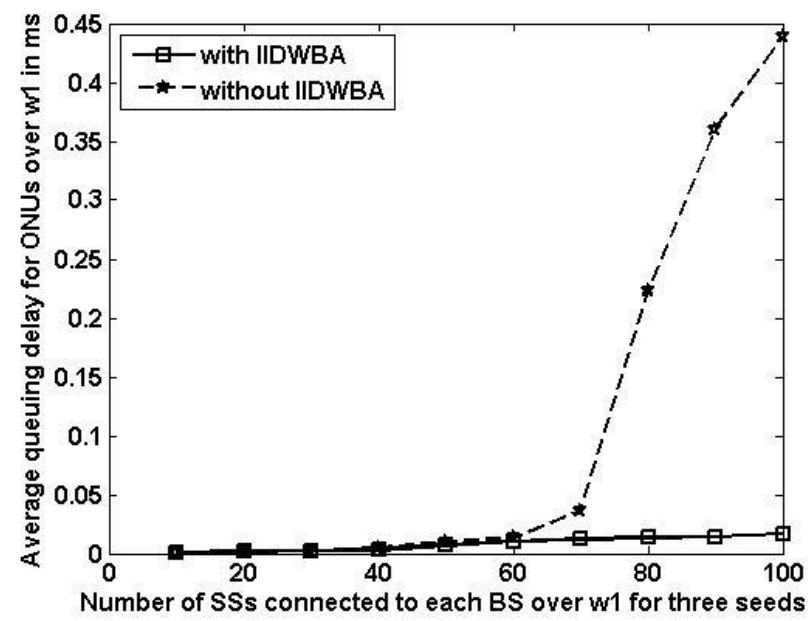

Figure 10 Average queuing delay for the ONUs on channel one, employing first traffic pattern

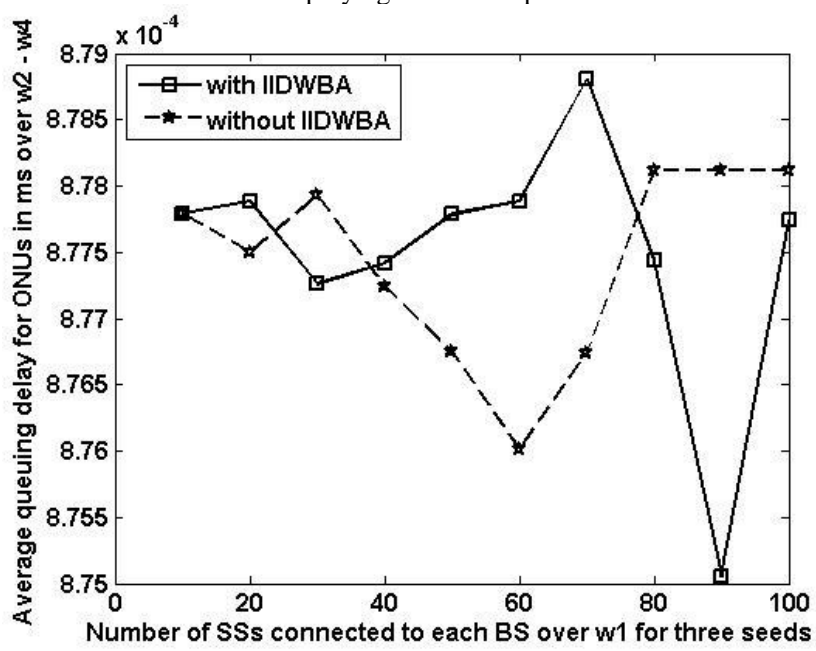

Figure 11 Average queuing delay for the ONUs associated with channel 2-4, employing first traffic pattern 


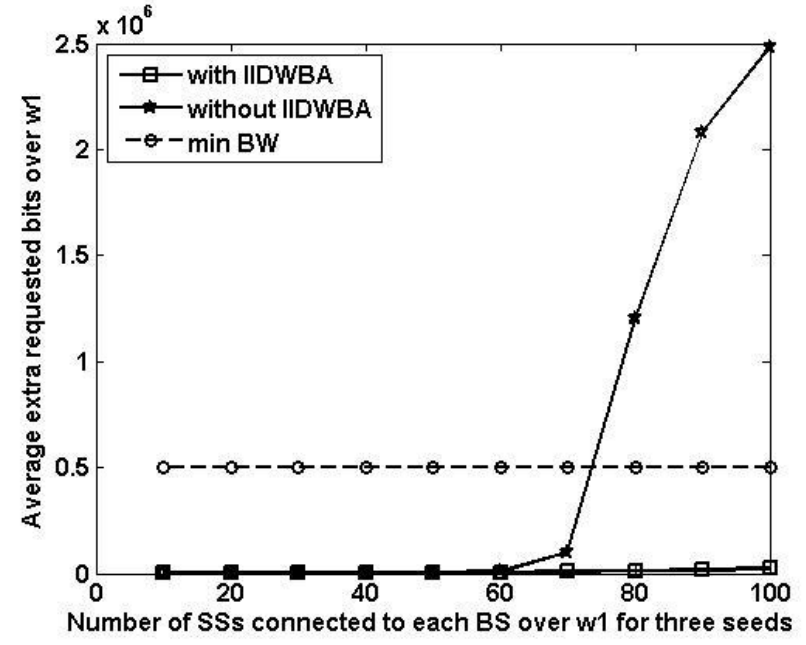

Figure 12 Average extra requested bandwidth from the ONUs associated with channel one, employing first traffic pattern

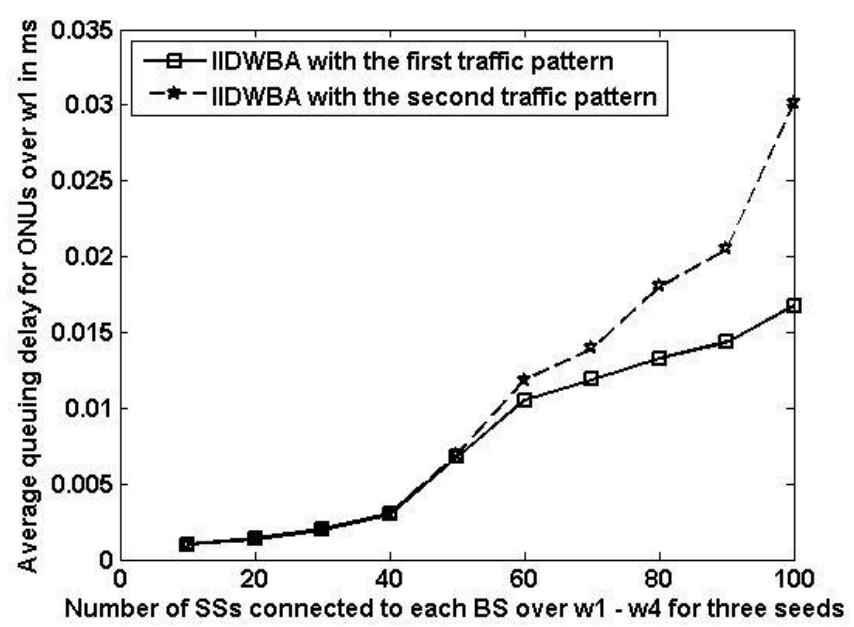

Figure 13 Average queuing delay for the ONUs associated with channel one employing the first and second traffic patterns

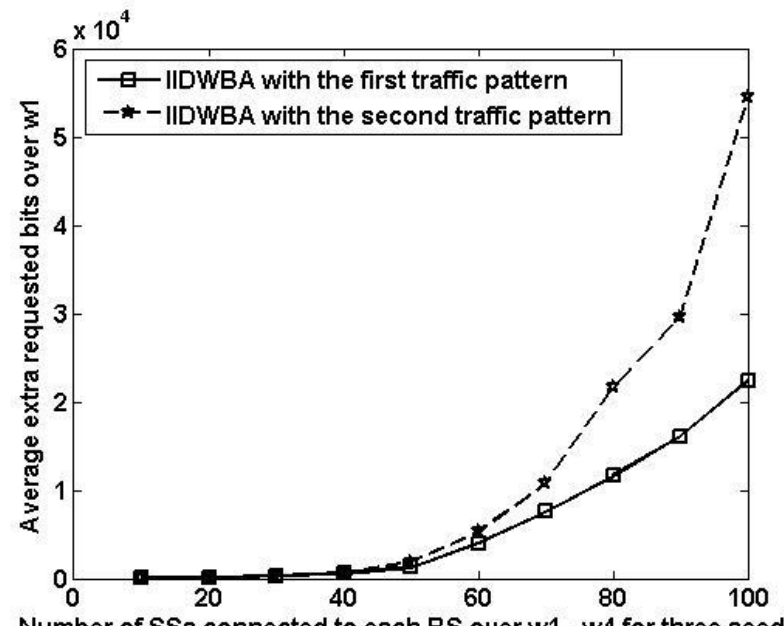

Number of SSs connected to each BS over w1 - w4 for three seeds

Figure 14 Average extra requested bandwidth from the ONUs associated with channel one employing the first and second traffic pattern as follow. When the second traffic pattern is employed, the load on all the channels starts building up gradually, therefore, the IIDWBA algorithm cannot find as much as excess bandwidth for the ONUs associated with $\mathrm{w}_{1}$ over $\mathrm{w}_{2}, \ldots, \mathrm{w}_{4}$ when it is compared to the first traffic pattern. This behaviour results in accumulating more packets in the queues associated with the ONUs over $\mathrm{w}_{1}$ thus longer queuing delay and larger average extra requested bits will be produced.

\section{CONCLUSION}

In this paper, an algorithm termed Inter-channel and Intrachannel Dynamic Wavelength/Bandwidth Allocation (IIDWBA) is proposed for the multi-channel PON integration with wireless technologies where the extra bandwidth from all the available channels associated with a given Server Station (SST) will be identified, collected, scheduled and allocated to the heavily loaded Client Stations (CSs) which may be scattered over the different channels in the same service cycle. The proposed algorithm works in three phases named Initialisation, Intra-channel bandwidth allocations and Interchannel bandwidth allocations. Through conducted simulation experiments, it is demonstrated that the proposed algorithm is capable of collecting the excess bandwidth from the lightly loaded channels and spreading them fairly across the heavily loaded CSs which may be scattered over different channels. The proposed algorithm showed a better performance when it was compared with the same simulation scenario but without employing the IIDWBA algorithm.

\section{REFERENCES}

[1] C. Xiao et al, "An Efficient Reservation MAC Protocol with Preallocation for High-Speed WDM-PON," IEEE INFOCOM Conf., Vol. 1, Aug. 2005, pp. 444 - 454.

[2] M. McGarry et al., "WDM Ethernet passive optical networks, "IEEE Optical Communications, Vo1.44, Issue2, Feb.2006, S18-S25.

[3] S. Ou et al., "Integrated Dynamic Bandwidth Allocation in Converged Passive Optical Networks and IEEE 802.16 Networks," IEEE Sys. J., Vol. 4, No. 4, Dec. 2010, pp. 467- 476.

[4] B. Skubic et al., "A Comparison of Dynamic Bandwidth Allocation for EPON, GPON, and Next-Generation TDM PON", IEEE Comu. Mag., March 2009, pp.S40-S48.

[5] G. Kramer et al., "Ethernet Passive OpticalNetwork (EPON): a missing link in an end-to-end optical internet," in Multi-Protocol Over WDM: Building the Next Generation Internet, S. Dixit, Ed. New York: Wiley, Mar. 2003.

[6] A. Banerjee et al., "Wavelength-division multiplexed passive optical network (WDM-PON) technologies for broadband access-A review [Invited]," OSA J. Opt. Netw.-Special Issue Optical Access Networks, vol. 4, no. 11, Nov. 2005, pp. 737-758.

[7] G. Shen et al, "Fixed Mobile Convergence Architectures for Broadband Access: Integration of EPON and WiMAX," IEEE Commun. Mag., Volume 45, Issue 8, August 2007, pp. 44 - 50.

[8] C.M. Assi et al., "Dynamic bandwidth allocation for Quality-of-Service over Ethernet PONs," IEEE J. Sel. Areas Commun., vol. 21, no. 9, Nov. 2003, pp. 1467-1477.

[9] OPNET Modeler 16.0, available at: www.opnet.com

[10] IEEE 802.3ah (EPON)at: http://www.ieee802.org/3/ah/index.html

[11] ITU-T G.9S4.x (GPON), at: http://www.itu.int/rec/T-REC-G/e

[12] ITU-T G.983.1 (BPON), at: http://www.itu.int/rec/T-REC-G.983.1200501-I/en.

[13] Y.Luo et al., "Resource Management for Broadband Access over TimeDivision Multiplexed Passive Optical Networks,” Sept.-Oct. 2007, vol. 21 , no. 5 , pp. $20-27$ 
[14] G. Kramer et al., "On Supporting Differentiated Classes of Service in Ethernet Passive Optical Networks," J. Opt. Netw., vol. 1, no. 8, pp. 280-298, 2002.

[15] T. Tang et al., "Performance Analysis of Bandwidth Allocation of Convergence of WiMAX and EPON," IEEE CS, 2009, pp. 662-665.

[16] Y.Luo et al., "Integrating Optical and Wireless Services in the Access Network," OSA, 2006.

[17] S. Sarkar et al., "Hybrid wireless-optical broadband- access network (WOBAN): A review of relevant challenges," JLT, Nov. 2007, Vol. 25, No. 11, pp. 3329-3340.

[18] K. Yang et al., "Convergence of Ethernet PON and IEEE S02.16 Broadband Access Networks and its QoS-Aware Dynamic Bandwidth Allocation Scheme," IEEE JSA, Feb. 2009, vol. 27, no.2, pp. 101-115.

[19] Z. Jia et aI., "Simultaneous generation of independent wired and wireless services using a single modulator in millimetre-wave-band radio-over-fibre systems," IEEE PTL., Oct. 2007,vol. 19, no.20, pp. 1691-1693.

[20] N. Moradpoor et al., "Hybrid Optical and Wireless technology Integrations for Next Generation Broadband Access Networks," IFIP/ IEEE BCN, May 2011, pp. 1013-1020.

[21] D.J. Shin et al., "Hybrid WDM/TDM-PON With Wavelength-SelectionFree Transmitters," JLT, Jan. 2005, 23: 1, pp. 187-194.

[22] S. Hussain et al., "EPON: an extensive review of up-to-date DBA Schemes", in Canadian Conf. on Electrical and Computer Eng, , Niagara Falls, ON, Canada, May, 2008, pp. 511-516,.

[23] G. Kramer et al., "Ethernet PON (ePON): Design and Analysis of an Optical Access Network," Photonic Network Commun., vol. 3. no. 3, July 2001 , pp. 307-19.

[24] H. Byun et al., "Dynamic Bandwidth Allocation Algorithm in Ethernet passive optical networks," IEE Elect. Lett., vol. 39, no. 13, June 2003, pp. 1001-1002.

[25] Y. Luo et al., "Limited Sharing with Traffic Prediction for Dynamic Bandwidth Allocation and QoS Provisioning over EPONs," J. Opt. Net., vol. 4, no. 9, Sep. 2005, pp. 561-572.

[26] IEEE P802.3av Task Force at: http://www.ieee802. org/3/av/tf_docs/10gepon_5criteria_0506.pdf

[27] C. Eklund et al., "IEEE standard 802.16: a technical overview of the WirelessMANTm air interface for broadband wireless access," IEEE Communications Magazine, 40, 2002, pp. 98-107.

[28] IEEE Standard for Local and Metropolitan Area Networks Part 16: Air Interface for Fixed Broadband Wireless Access Systems, 2004.

[29] IEEE Standard for Local and Metropolitan Area Networks, Part 16: Air Interface for Fixed and Mobile Broadband Wireless Access SystemsAmendment 2: Physical and Medium Access Control Layers for Combined Fixed and Mobile Operation in Licensed Bands and Corrigendum 1. IEEE Computer Society and the IEEE Microwave Theory and Techniques Society Feb. 2006.

[30] Y. Luo et al., "QoS-aware scheduling over hybrid optical wireless networks," in Proc. OFC/NFOEC 2007, Anaheim, CA, Mar. 2007, pp. $1-7$.

[31] Y. Kun et al., "Convergence of Ethernet PON and IEEE 802.16 broadband access networks and its QoS-aware dynamic bandwidth allocation scheme," IEEE J. Sel. Areas Commun., vol. 27, no. 2, 2009, pp. 101-116.

[32] W.-T. Shaw et al., "Hybrid Architecture and Integrated Routing in a Scalable Optical-Wireless Access Network," JLT. vol. 25, no. 11, Nov. 2007, pp. 3443-3451.

[33] C. Ranaweera et al., "Quality of Service Assurance in EPON-WiMAX Converged Network", IEEE MWP, 2011, pp. 369-372.

[34] A. Ahmed et al., "A New Bandwidth Allocation Algorithm for EPONWiMAX Hybrid Access Networks," IEEE Globecom, 2010.

[35] A. R. Dhaini et al., "MC-FiWiBAN an Emergency-Aware MissionCritical Fiber-Wireless Broadband Access Network," IEEE Commun. Mag., Jan. 2011, pp. 134-142.

[36] I.-S. Hwang et al., "Advanced Dynamic Bandwidth Allocation and Scheduling Scheme for the Integrated Architecture of EPON and WiMAX," 2009, pp. 655-660.

[37] B. Jung et al., "Centralized Scheduling Mechanism for Enhanced Endto-End Delay and QoS Support in Integrated Architecture of EPON and WiMAX”, JLT, vol. 28, no. 16, Aug.2010.
[38] F- T. An et al., "A new Dynamic Bandwidth Allocation Protocol with Quality of Service in Ethernet-based Passive Optical Networks," in Proc. International Conference on Wireless and Optical Communication (WOC), Banff, Canada, Jul. 2003.

[39] G. Kramer, "Ethernet Passive Optical Networks," New York, McGrawHill, 2005.

[40] "Specifications of the optical components," available at: http://www.huihongfiber.com

[41] Y. H. Min, M. H. Lee, J. J. Ju, S. K. Park, and J. Y. Do, "Polymeric 16 16 arrayed-waveguide grating router using fluorinated polyethers operating around $1550 \mathrm{~nm}$," IEEE Journal on Selected Topics Quantum Electron., vol. 7, no. 5, September 2001, pp. 806-811.

[42] S. Hassanpour, B. Asadi, Y. Vejdanparast, P. Zargar, "Improving reliability of routing in Wireless Body Area Sensor Networks using Genetic Algorithm," IEEE International Conference on Computer Science and Automation Engineering (CSAE), vol. 2, June 2011, pp $590-593$. 